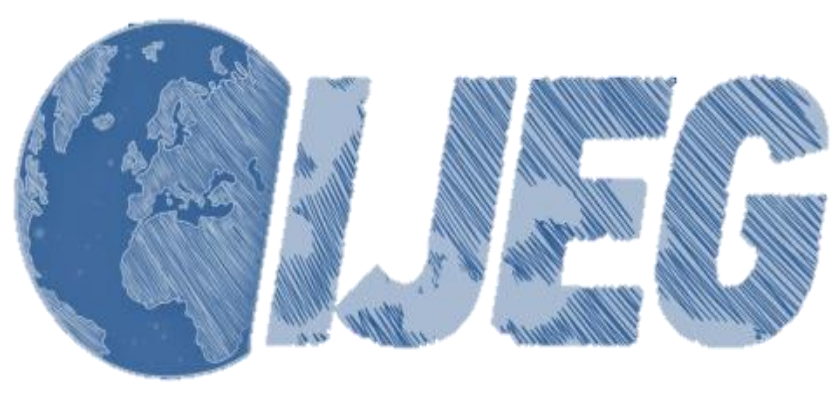

International Journal of Engineering and Geosciences (IJEG), Vol; 5, Issue; 2, pp. 073-093, June, 2020, ISSN 2548-0960, Turkey, DOI: $10.26833 /$ ijeg.580027

\title{
PERFORMANCE ANALYSIS OF AMBIGUITY RESOLUTION ON PPP AND RELATIVE POSITIONING TECHNIQUES: CONSIDERATION OF SATELLITE GEOMETRY
}

\author{
Sermet Ogutcu* \\ Necmettin Erbakan University, Engineering Faculty, Department of Geomatic Engineering, Konya, Turkey \\ (sermetogutcu@konya.edu.tr); ORCID 0000-0002-2680-1856
}

*Corresponding Author, Received: 19/06/2019, Accepted: 28/08/2019

\begin{abstract}
Ambiguity resolution plays an important role in surveying using Precise Point Positioning (PPP) and relative positioning techniques that require high accuracy. In this study, ambiguity resolution performance of PPP and relative positioning under the unobstructed (with $7^{\circ}$ cut-off angle) and constrained environment (with $25^{\circ}$ cut-off angle, such as nearby buildings and street-canyons) using final/ultra-rapid orbit and clock products are investigated for different observation time. Seventeen globally distributed stations and six baselines of lengths from $270 \mathrm{~km}$ to $2100 \mathrm{~km}$ are chosen for conducting PPP and relative positioning, respectively. A 31-day period in January 2018 is chosen for processing using 24-, 12-, 6-, 4-, 2- and 1-h observations. The results indicate that sub-mm to $\mathrm{cm}$ levels of improvement in horizontal and vertical coordinate components are generally observed with ambiguity resolution for PPP and relative positioning techniques compared to the float counterparts. Moreover, accuracy degradation of ambiguity resolution compared to float solution is observed generally in the vertical component using the $25^{\circ}$ elevation cut-off angle for both techniques. As the observation time increases, the accuracy improvements from ambiguity resolution decrease for each technique. In addition, fixing to the wrong integer ambiguities are generally seen with a short observation time and a $25^{\circ}$ elevation cut-off angle for both techniques due to the poor satellite geometry. As far as baseline length in relative technique is concerned, the testing results show that there is no direct relation between baseline length and the accuracy improvement from ambiguity resolution compared to the float solution. The results also reveal that the coordinates obtained from ambiguity resolution does not significantly change in the relative technique using final or ultra-rapid orbit/clock products, whereas the changes in PPP are significant for most of the stations.
\end{abstract}

Keywords: Ambiguity Resolution, GPS, Precise Point Positioning, Relative Positioning, Satellite Geometry 


\section{INTRODUCTION}

Global Navigation Satellite System (GNSS) has been commonly used in several scientific and engineering applications including positioning, navigation, and time transfer for several decades. Relative positioning and Precise Point Positioning (PPP) are two main techniques for geodetic surveying. Relative positioning techniques are based on the principle that at least one of the receivers serves as a base station with known coordinates and the other receivers serve as rover stations, in which the coordinates are determined relative to the base station (Blewitt and Young 1989; Schwarz et al. 2009).

PPP (Zumberge et al. 1997) has become a powerful tool for obtaining precise positioning using precise satellite orbit and clock corrections provided by, for example, International GNSS service. It plays a key role in much scientific research, such as tectonic, geophysical studies, structural health monitoring and, (Shi et al. 2010; Geng et al. 2017; Alcay et al. 2019) atmosphere and earthquake monitoring (Bar-Server et al. 1998; Li et al. 2013).

PPP and relative methods have advantages and drawbacks. While relative technique requires as minimum of two GNSS receivers, PPP requires a single GNSS receiver. In this way, field operational cost can be significantly reduced using PPP. Even though millimeter level accuracies can be achieved when processing daily solutions in static mode using both techniques, the long convergence time restricts PPP users to short observation sessions or real-time applications (Pehlivan et al. 2019; Yigit et al. 2014). Moreover, the accuracy of coordinates obtained from traditional PPP is still weaker than that obtained from the relative techniques for short observation time, since traditional PPP is mostly implemented with an ambiguity-float solution. The main obstacle that prevents PPP ambiguity resolution (AR) is the non-integer uncalibrated hardware delay (UHD) originating both in receivers and satellites, which is eliminated by double differencing in relative positioning (Geng et al. 2009). Implementing AR in PPP requires determining UHD in advance using a network. In this way, AR in PPP is only possible when service providers run the network consisting of reference stations to compute hardware biases, in addition to the satellite orbits and clocks that are consistent for PPP AR (Teunissen and Khodabandeh 2015; Choy et al. 2017). Most of the online PPP service providers such as GAPS, CRSR-PPP (transition process to AR started on August 16th, 2018), MagicGNSS, and several types of PPP software such as Bernese, RTKLIB, and GpsTools provide the ambiguityfloat solution.

There are numerous research studies addressing AR in PPP. Goudarzi et al. (2018) investigated the performance of PPP with and without AR to recover station velocities using daily observations. They found that the estimated horizontal and vertical velocities from the two solutions were in good agreement. Gandolfi et al. (2017) assessed the effect of GPS-only PPP-AR on coordinate precision using one year of data from 14 European GNSS stations. They found that precision only for the easting component obtained from fixed solutions significantly changed compared to the float solutions. Xiao et al. (2018) investigated the GPS-only AR in PPP using 10 days of data with 3-h sessions for 20 stations. They found that root-mean-squared-error (RMSE) of the horizontal components obtained from the fixed solutions is significantly lower compared to the float solutions. $\mathrm{Li}$ et al. (2018) evaluated the performance of kinematic GPS-only PPP-AR under different cut-off angles (7-30) and different session lengths (from 10 min to 2 -hours). They found that as the degree of elevation mask increased, the accuracy of GPS-only PPP-AR solution decreased dramatically. Xin et al. (2018) investigated BeiDou-only PPP-AR with two local networks (Hong Kong, CMONOC) and one global network (MGEX) using 10 days of data. They found that after AR, the improvements in two-dimensional and vertical positioning of $1 \mathrm{~h}$ static PPP were $1.8 \mathrm{~cm} / 4.5 \mathrm{~cm}, 8.6$ $\mathrm{cm} / 4.5 \mathrm{~cm}$ and $1.7 \mathrm{~cm} / 3.1 \mathrm{~cm}$ for the Hong Kong, CMONOC, and MGEX networks respectively. Geng et al. (2009) conducted GPS-only PPP- AR using six days of data with 27 IGS stations. The results show that hourly position accuracy is improved from $3.8 \mathrm{~cm}, 1.5 \mathrm{~cm}$, and $2.8 \mathrm{~cm}$ to $0.5 \mathrm{~cm}, 0.5 \mathrm{~cm}$ and $1.4 \mathrm{~cm}$ in east, north, and up respectively.

There are also studies related to $\mathrm{AR}$ in relative positioning. Chen et al. (2017) investigated the repeatability of short baseline components for float and fixed solutions using $24 \mathrm{~h}$ data. They found $2.8 \mathrm{~mm}$ and $5 \mathrm{~mm}$ improvements for horizontal and vertical components, respectively. Brach and Zasada, 2014 conducted relative positioning using fixed and float solutions under forest conditions. The experimental results show that a relatively small number of fixed solutions were obtained under forest conditions compared to open sky conditions. The results also confirmed that by extending the float observation time, it is possible to achieve accuracy similar to the fixed solution in the forest conditions. Bezcioglu et al. (2019) conducted kinematic PPP-AR in Antarctic for the first time. They found that PPP-AR produced more accurate results than PPP-float, with a faster convergence.

The above-mentioned studies were mainly conducted using the fixed elevation cut-off angles to obtain an unobstructed observation environment (around $7^{\circ}-10^{\circ}$ ) and final orbit/clock products. Coordinate improvement and reliability of fixing ambiguities after AR for PPP and relative positioning under poor satellite geometry (such as nearby buildings and street-canyons) and using ultrarapid orbit and clock products taking into consideration of the observing sessions (for near-real-time users) are not well addressed in the current literature, and worth investigating. High success rates are required for AR to be reliable. Unsuccessful AR, when passed unnoticed, often leads to unacceptable errors in the positioning results. In this paper, AR performance of PPP and relative positioning under the constrained environment and using final/ultra-orbit and clock products are investigated.

This paper is organized as follows. Section 2 describes the PPP and relative positioning functional models, Section 3 presents the data processing. The results are summarized and discussed in Section 4 and the work is concluded in Section 5. 


\section{FUNCTIONAL MODELS OF PPP AND RELATIVE POSITIONING}

In general, ionosphere-free code and carrier phase observations are used for PPP and relative positioning in order to eliminate the first-order ionosphere effect. The ionosphere-free code and carrier phase observables can be written as:

$$
\begin{aligned}
& P_{I F, r}^{S}=\left(f 1^{2} * P 1^{s}-f 2^{2} * P 2^{s}\right) /\left(f 1^{2}-f 2^{2}\right) \\
& \emptyset_{I F, r}^{s}=\left(f 1^{2} * \emptyset 1^{s}-f 2^{2} * \emptyset 2^{s}\right) /\left(f 1^{2}-f 2^{2}\right)
\end{aligned}
$$

where $\mathrm{f} 1$ and $\mathrm{f} 2$ are two carrier-phase frequencies in Hertz and P1, P2, $\varnothing 1$, and $\varnothing 1$ are the measured pseudorange and carrier phase in meters on two frequencies. The observation equations can be written for PPP as follows:

$$
\begin{aligned}
& P_{I F, r}^{S}=\rho+c *\left(d t_{r}-d t^{s}\right)+d_{\text {trop }}+H D_{P, r, I F}- \\
& H D^{P, S, I F}+\epsilon_{P, I F} \\
& \emptyset_{I F, r}^{S}=\rho+c *\left(d t_{r}-d t^{s}\right)+d_{t r o p}+\lambda_{I F} *\left(N_{I F}^{S}+\right. \\
& \left.U H D_{I F, r}-U H D^{I F, s}\right)+\epsilon_{\emptyset, l f}
\end{aligned}
$$

where the superscript $\mathrm{s}$ represents the satellite, the subscript $\mathrm{r}$ represents the receiver, $P_{I F, r}^{S}$ and $\quad \emptyset_{I F, r}^{S}$ are the ionosphere-free combination of code and phase observations, $\rho$ is the geometric range in meters, $\mathrm{c}$ is the speed of light in meters per second, $d t_{r}$ is the receiver clock offset in seconds, $d t^{s}$ is the satellite clock offset in seconds, $d_{\text {trop }}$ is the tropospheric delay in meters, $\lambda_{I F}$ is the ionosphere-free wavelength, $N_{I F}$ is the ionospherefree phase initial ambiguity in cycle, and $U H D_{I F, r}$ and $U H D^{I F, S}$ are the ionosphere-free uncalibrated hardware delays in the cycle originating both in receivers and satellites. $H D_{P, r, I F}$ and $H D^{P, S, I F}$ are the ionosphere-free receiver and satellite code hardware delays and $\epsilon_{P, I F}$ and $\epsilon_{\emptyset, I F}$ represent the ionosphere-free code and phase measurement noise. Ionosphere-free wavelength, ambiguity, code hardware delay and UHD can be written as:

$$
\begin{aligned}
& \lambda_{I F}=\frac{f 1^{2}}{f 1^{2}-f 2^{2}} * \lambda_{1}-\frac{f 2^{2}}{f 1^{2}-f 2^{2}} * \lambda_{2} \\
& N_{I F}=\frac{f 1^{2} * N_{1}}{f 1^{2}-f 2^{2}}-\frac{f 2^{2} * N_{2}}{f 1^{2}-f 2^{2}} \\
& H D_{P, r, I F}=\left(f 1^{2} * H D_{r, 1}-f 2^{2} * H D_{r, 2}\right) /\left(f 1^{2}-f 2^{2}\right) \\
& (7) \\
& H D^{P, s, I F}=\left(f 1^{2} * H D^{s, 1}-f 2^{2} * H D^{s, 2}\right) /\left(f 1^{2}-f 2^{2}\right) \\
& (8) \\
& U H D_{I F, r}=\left(f 1^{2} * U H D_{r, 1}-f 2^{2} * U H D_{r, 2}\right) /\left(f 1^{2}-\right. \\
& \left.f 2^{2}\right) \\
& U H D^{I F, s}=\left(f 1^{2} * U H D^{s, 1}-f 2^{2} * U H D^{s, 2}\right) /\left(f 1^{2}-\right. \\
& \left.f 2^{2}\right)
\end{aligned}
$$

As is seen in Equation 4, ionosphere-free ambiguity can only be estimated as a float (real-value) unknown unless using UHD information from the network. For PPP users, the receiver dependent UHD is generally not a concern and can be safely ignored ( $\mathrm{Li}$ et al. 2016).

The functional model in relative positioning uses double-differenced (DD) observables, and functional equations can be written as follows (Wielgosz, 2011);

$$
\begin{aligned}
& \emptyset_{1, i j}^{k l}=\rho_{i j}^{k l}-T_{i j}^{k l}+I_{i j}^{k l}-\lambda_{1} * N_{1, i j}^{k l}+\epsilon_{\emptyset, 1, i j}^{k l} \\
& \text { (11) } \\
& \emptyset_{2, i j}^{k l}=\rho_{i j}^{k l}-T_{i j}^{k l}+\left(\frac{f 1^{2}}{f 2^{2}}\right) * I_{i j}^{k l}-\lambda_{2} * N_{2, i j}^{k l}+\epsilon_{\emptyset, 2, i j}^{k l} \\
& \text { (12) } \\
& P_{1, i j}^{k l}=\rho_{i j}^{k l}-T_{i j}^{k l} \quad-\quad I_{i j}^{k l} \quad+\quad \epsilon_{P, 1, i j}^{k l} \\
& \text { (13) } \\
& P_{2, i j}^{k l}=\rho_{i j}^{k l}-T_{i j}^{k l} \quad-\quad\left(\frac{f 1^{2}}{f 2^{2}}\right) * I_{i j}^{k l}+\epsilon_{P, 2, i j}^{k l}
\end{aligned}
$$

where $\mathrm{i}$ and $\mathrm{j}$ are the receiver indices, $\mathrm{k}$ and $\mathrm{l}$ the satellite indices, $\emptyset_{n, i j}^{k l}$ and $P_{n, i j}^{k l}$ are the DD phase and code observations on frequency $\mathrm{n}(\mathrm{n}=1,2), \rho_{, i j}^{k l}$ is the DD geometric distance between receiver and satellite, $T_{i j}^{k l}$ is the DD tropospheric delay, $I_{i j}^{k l}$ is DD ionospheric delay and $N_{n, i j}^{k l}$ is the DD carrier phase ambiguities on two frequencies, $\epsilon_{\emptyset, n, i j}^{k l}$ and $\epsilon_{P, n, i j}^{k l}$ are the DD phase and code measurements noise. The unknown parameters are receiver three-dimensional coordinates, DD ionospheric delay and DD ambiguities. Receiver and satellite clock biases are removed using the DD combination, whereas in PPP, receiver clock bias should be estimated as an unknown and satellite clock bias should be obtained from an external source such as IGS clock products. Another advantage of DD combination is that the ambiguity term preserves its integer nature.

Obtaining the fixed solution is generally done in three sequential steps. In the first step, the unknown parameters are solved without taking into account the integer ambiguity. This result is often referred to as the float solution. In the second step, the float solution is used to estimate the integer ambiguity. In the last step, the computed integer ambiguities are used to improve the unknown parameters in the float solution (Joosten and Tiberius, 2000). The unknown parameters are recomputed with the ambiguities constrained to the integer values. This recomputed process is generally referred to in GNSS processing software as the iteration.

There are several techniques to compute the integer value of ambiguities. Wide-lane and narrow-lane combinations (Teunissen and Montenbruck, 2017) are commonly used in the GNSS community, and one of the subjects of this study. The equations for phase and code observations can be written as follows; 
$\emptyset_{L W}=\left(f_{1} * \emptyset_{1}-f_{2} * \emptyset_{2}\right) /\left(f_{1}-f_{2}\right)$

$\emptyset_{L N}=\left(f_{1} * \emptyset_{1}+f_{2} * \emptyset_{2}\right) /\left(f_{1}+f_{2}\right)$

$P_{L W}=\left(f_{1} * P_{1}-f_{2} * P_{2}\right) /\left(f_{1}-f_{2}\right)$

$P_{L N}=\left(f_{1} * P_{1}+f_{2} * P_{2}\right) /\left(f_{1}+f_{2}\right)$

where $\emptyset_{L W} / \emptyset_{L N}$ and $P_{L W} / P_{L N}$ are wide-lane/narrowlane phase and code combinations respectively, with corresponding wavelengths of $\sim 0.86 \mathrm{~m}$ and $\sim 0.107 \mathrm{~m}$ for wide-lane and narrow-lane combinations. Ionospherefree ambiguity is usually expressed as wide- and narrowlane ambiguity fixing. The wide- and narrow-lane ambiguities cannot be estimated and fixed simultaneously. The main reason is that the equation corresponding to the narrow-lane ambiguity contains the wide-lane ambiguity. Usually, ambiguities are resolved by first computing wide-lane and then narrow-lane ambiguities. An ionosphere-free ambiguity can be fixed only if both its wide- and narrow-lane ambiguities are fixed (Ge et al. 2008).
Ambiguity resolution in PPP was conducted using JPL orbit and clock products which provide wide-lane and dual-frequency phase bias information from the global network of GPS receivers that were used to generate the orbit and clock solutions for the GPS satellites (Bertiger et al., 2010).

\section{DATA PROCESSING}

Seventeen globally distributed stations and six baselines of lengths varying from $270 \mathrm{~km}$ to $2100 \mathrm{~km}$ were chosen for conducting PPP and relative positioning, respectively. Figure 1 and Table 1 show the stations and the baselines used for PPP and the relative positioning processing respectively. First stations in Table 1 were used as fiducial stations. To form varying lengths of baselines for relative positioning, most of the used stations are different for PPP and relative positioning.

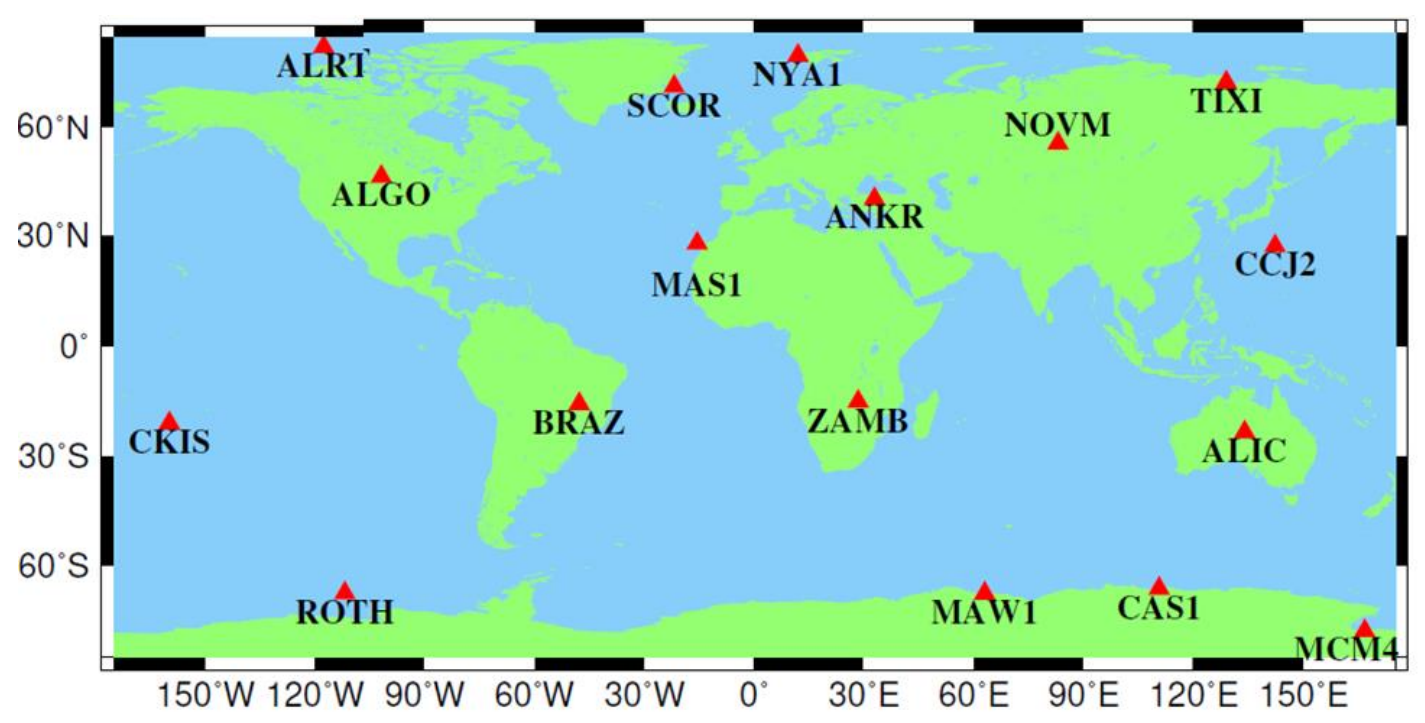

Figure 1. Stations used for PPP processing

Table 1. Baselines

\begin{tabular}{|c|c|c|c|}
\hline Baselines & $\begin{array}{c}\text { Length } \\
(\mathbf{k m})\end{array}$ & $\begin{array}{c}\text { Mean } \\
\text { latitude }\end{array}$ & $\begin{array}{c}\text { Mean } \\
\text { longitude }\end{array}$ \\
\hline $\begin{array}{c}\text { MERS- } \\
\text { MEDI }\end{array}$ & 2100 & 40.3 & 23 \\
\hline $\begin{array}{c}\text { ALRT- } \\
\text { NYA1 }\end{array}$ & 1285 & 80.7 & -25 \\
\hline $\begin{array}{c}\text { VIS0- } \\
\text { MDVJ }\end{array}$ & 1100 & 56.8 & 28 \\
\hline $\begin{array}{c}\text { GANP- } \\
\text { LAMA }\end{array}$ & 536 & 51.4 & 20 \\
\hline $\begin{array}{c}\text { PALM- } \\
\text { ROTH }\end{array}$ & 362 & -66.2 & -66 \\
\hline $\begin{array}{c}\text { MARS- } \\
\text { IENG }\end{array}$ & 270 & 44.1 & 89 \\
\hline
\end{tabular}

The 31-day period of January 2018 was chosen for PPP and relative processing. Daily RINEX files of the stations were subdivided into mutually non-overlapping sessions as in 24-12-6-4-2-1 h subsets. Data integrity of the RINEX files was checked using in-house software. Data integrities of less than $99 \%$ were removed from the processing. Eight daily RINEX files were discarded from the dataset.

Satellite elevation cut-off angles of $7^{\circ}$ and $25^{\circ}$ were chosen to represent the open sky and obstructed conditions such as street-canyons. Final and ultra-rapid satellite orbit/clock products (observed part) were used to investigate the ambiguity resolution impact on the estimated coordinates for post-processing and near-realtime users (Tusat et al. 2018). Each data set was processed 
by GAMIT/GLOBK and GIPSY/OASIS scientific software for relative and PPP techniques respectively.
Table 2 summarizes the processing parameters for each type of software.

Table 2. Processing parameters

\begin{tabular}{|c|c|c|}
\hline Processing parameters & Relative processing strategy & PPP processing strategy \\
\hline GNSS system & GPS & GPS \\
\hline Observations & Phase and code data on two frequencies & Phase and code data on two frequencies \\
\hline $\begin{array}{l}\text { Data weight of linear } \\
\text { combination phase and code }\end{array}$ & $1 \mathrm{~cm} / 1 \mathrm{~m}$ & $1 \mathrm{~cm} / 1 \mathrm{~m}$ \\
\hline Weighting with elevation & Applied & Applied \\
\hline Sampling interval & $30 \mathrm{~s}$ & $30 \mathrm{~s}$ \\
\hline Cut-off angle & $7^{\circ} / 25^{\circ}$ & $7^{\circ} / 25^{\circ}$ \\
\hline Satellite orbit and clock & IGS final/ultra-rapid & JPL final/ultra-rapid (fiducial frame) \\
\hline Relativistic corrections & Removed using double differences & $\begin{array}{l}\text { Periodic clock corrections and gravitational } \\
\text { bending (shapiro delay) were applied }\end{array}$ \\
\hline Receiver clock error & Removed using double differences & Estimated as white noise \\
\hline Reference frame realization & $\begin{array}{l}\text { Tight constraint using the fiducial } \\
\text { station's true coordinates (IGS14) from } \\
\text { IGS weekly combined solutions }\end{array}$ & $\begin{array}{c}\text { Fix to satellites ECEF coordinates of JPL } \\
\text { fiducial frame (IGS14) }\end{array}$ \\
\hline Cycle-slip & $\begin{array}{c}\text { Corrected by wide-lane combination } \\
\text { using phase and code data }\end{array}$ & Corrected by Melborne-Wubbena combination. \\
\hline Receiver clock jump & Corrected & Corrected \\
\hline Ionosphere & $\begin{array}{l}\text { The first order is eliminated by IF } \\
\text { combination. Second order is removed } \\
\text { using IGS IONEX file }\end{array}$ & $\begin{array}{c}\text { The first order is eliminated by IF combination. } \\
\text { Second order is removed using JPL IONEX } \\
\text { file. }\end{array}$ \\
\hline A Priori troposphere & $\begin{array}{l}\text { GPT2 model (Lagler et al. 2013) were } \\
\text { applied using tropospheric gradient }\end{array}$ & $\begin{array}{l}\text { GPT2 model (Lagler et al. 2013) were applied } \\
\text { using tropospheric gradient. }\end{array}$ \\
\hline Wet tropospheric delay & $\begin{array}{l}\text { Estimated as random-walk model }\left(5 \times 10^{-}\right. \\
\left.{ }^{8} \mathrm{~m}^{2} / \mathrm{s}\right)\end{array}$ & Estimated as random-walk model $\left(5 \times 10^{-8} \mathrm{~m}^{2} / \mathrm{s}\right)$ \\
\hline Tropospheric gradients & $\begin{array}{l}\text { Estimated as random-walk model }\left(5 \times 10^{-}\right. \\
\left.{ }_{8} \mathrm{~m}^{2} / \mathrm{s}\right)\end{array}$ & Estimated as random-walk model $\left(5 \times 10^{-8} \mathrm{~m}^{2} / \mathrm{s}\right)$ \\
\hline Phase ambiguity & $\begin{array}{l}\text { Ambiguity resolution using wide- and } \\
\text { narrow-lane and additionally float } \\
\text { estimation }\end{array}$ & $\begin{array}{l}\text { Ambiguity resolution using wide- and narrow- } \\
\text { lane and additionally float estimation. }\end{array}$ \\
\hline Adjustment model & Stochastic Kalman filter & $\begin{array}{l}\text { Stochastic Kalman filter/smoother implemented } \\
\text { as square root information filter with smoother }\end{array}$ \\
\hline $\begin{array}{c}\text { Satellite/receiver antenna phase } \\
\text { offset }\end{array}$ & $\begin{array}{l}\text { PCO and PCV values from up-to-date } \\
\text { igs.atx were used }\end{array}$ & $\begin{array}{l}\text { PCO and PCV values from up-to-date igs.atx } \\
\text { were used }\end{array}$ \\
\hline Tidal effects & $\begin{array}{c}\text { Solid tides, ocean tide loading and polar } \\
\text { tides (IERS 2010; Petit and Luzum, } \\
2010 \text { ) }\end{array}$ & $\begin{array}{l}\text { Solid tides, ocean tide loading and polar tides } \\
\quad \text { (IERS 2010; Petit and Luzum, 2010) }\end{array}$ \\
\hline Wind-up effect & Corrected (Wu et al. 1992) & Corrected (Wu et al. 1992) \\
\hline Satellite DCB & $\begin{array}{l}\text { Corrected by up to date CODE DCB } \\
\text { product }\end{array}$ & Corrected by up to date CODE DCB product. \\
\hline Eclipse strategy & $\begin{array}{l}\text { Eclipsing satellites were not used until } \\
\text { satellites reach nominal attitude }\end{array}$ & $\begin{array}{l}\text { Eclipsing satellites were not used until satellites } \\
\text { reach nominal attitude. }\end{array}$ \\
\hline
\end{tabular}

\section{ANALYSIS AND RESULTS}

The reference coordinates of the stations were taken from IGS weekly combined solutions with an accuracy of within a few millimeters. If any coordinate component's error in regard to the true solution was greater than $30 \mathrm{~cm}$, it was assumed to be an outlier and removed from the RMSE computation. This is an arbitrary assumption based on the range of errors and is a suitable criterion with which to determine a threshold without making other outlier assumptions, which sometimes are not suitable (Gandolfi et al. 2017). AR impacts on the estimated coordinates were investigated as the coordinates' improvement with regards to the float solutions in horizontal and vertical components. The positive improvement denotes that RMSE of fix solution is smaller compared to float solution. The negative improvement denotes that RMSE of float solution is smaller compared to fix solution. In order to provide a better visualization. Tables 3 and 4 show the RMSEs of fixed and float solutions with final products using $7^{\circ} / 25^{\circ}$ cut-off angles $\left(\sigma \_n, \sigma \_\right.$e, $\left.\sigma \_u\right)$ for PPP. Tables 5 and 6 show the same statistics for relative positioning as in the PPP results, but RMSEs of ultra-products were not given due to the nearly identical results compared to the final product. Figure 2-5 show the PPP horizontal and vertical RMSE improvements of fixed solution with respect to float solutions for final and ultra-products using $7^{\circ} / 25^{\circ}$ cut-off angles. The same statistical values were given in Figure 6-7 for relative positioning techniques except for ultra-product due to the nearly identical results with respect to final product. 
International Journal of Engineering and Geosciences (IJEG),

Vol; 5, Issue; 2, pp. 073-093, February, 2020,

Table 3. RMS of stations used for PPP processing for 24-12-6 $\mathrm{h}$ (values are in $\mathrm{mm}$ )

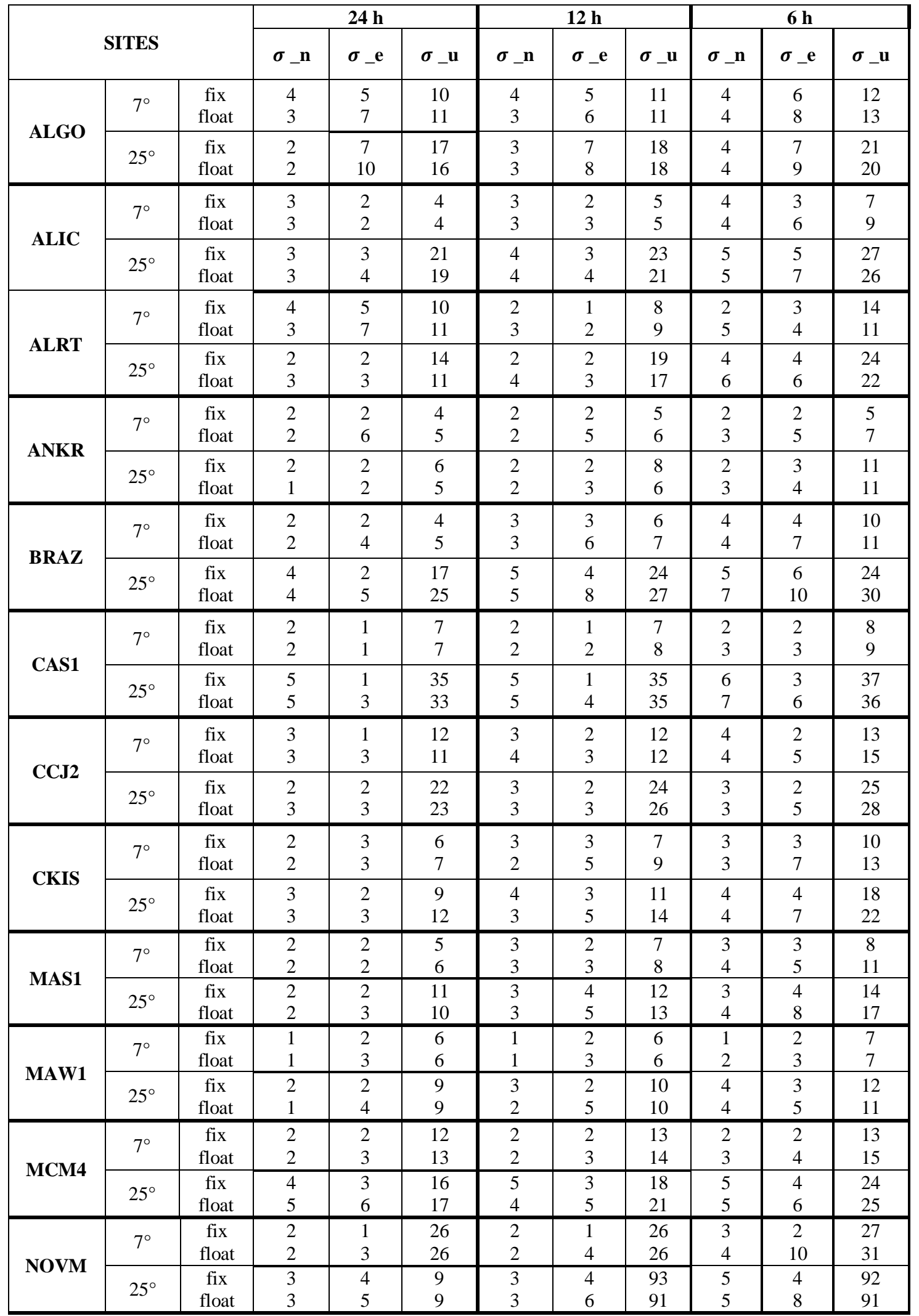


International Journal of Engineering and Geosciences (IJEG),

Vol; 5, Issue; 2, pp. 073-093, February, 2020,

Table 3 (Continued)

\begin{tabular}{|c|c|c|c|c|c|c|c|c|c|c|c|}
\hline \multirow{2}{*}{ NYA1 } & $7^{\circ}$ & $\begin{array}{c}\text { fix } \\
\text { float }\end{array}$ & $\begin{array}{l}2 \\
2 \\
\end{array}$ & $\begin{array}{l}3 \\
3 \\
\end{array}$ & $\begin{array}{l}4 \\
6 \\
\end{array}$ & $\begin{array}{l}2 \\
2 \\
\end{array}$ & $\begin{array}{l}3 \\
3 \\
\end{array}$ & $\begin{array}{l}5 \\
6 \\
\end{array}$ & $\begin{array}{l}2 \\
3 \\
\end{array}$ & $\begin{array}{l}3 \\
4 \\
\end{array}$ & $\begin{array}{l}7 \\
9 \\
\end{array}$ \\
\hline & $25^{\circ}$ & $\begin{array}{c}\text { fix } \\
\text { float }\end{array}$ & $\begin{array}{l}1 \\
2 \\
\end{array}$ & $\begin{array}{l}2 \\
3 \\
\end{array}$ & $\begin{array}{l}40 \\
37 \\
\end{array}$ & $\begin{array}{l}2 \\
2 \\
\end{array}$ & $\begin{array}{l}3 \\
3 \\
\end{array}$ & $\begin{array}{l}40 \\
38 \\
\end{array}$ & $\begin{array}{l}2 \\
3 \\
\end{array}$ & $\begin{array}{l}3 \\
4 \\
\end{array}$ & $\begin{array}{l}42 \\
40 \\
\end{array}$ \\
\hline \multirow{2}{*}{ ROTH } & $7^{\circ}$ & $\begin{array}{c}\text { fix } \\
\text { float }\end{array}$ & $\begin{array}{l}2 \\
2 \\
\end{array}$ & $\begin{array}{l}2 \\
3 \\
\end{array}$ & $\begin{array}{l}4 \\
5 \\
\end{array}$ & $\begin{array}{l}3 \\
2 \\
\end{array}$ & $\begin{array}{l}2 \\
3 \\
\end{array}$ & $\begin{array}{l}5 \\
5 \\
\end{array}$ & $\begin{array}{l}3 \\
3 \\
\end{array}$ & $\begin{array}{l}2 \\
4 \\
\end{array}$ & $\begin{array}{l}7 \\
6 \\
\end{array}$ \\
\hline & $25^{\circ}$ & $\begin{array}{c}\text { fix } \\
\text { float }\end{array}$ & $\begin{array}{l}2 \\
2 \\
\end{array}$ & $\begin{array}{l}2 \\
3 \\
\end{array}$ & $\begin{array}{l}36 \\
36 \\
\end{array}$ & $\begin{array}{l}3 \\
3 \\
\end{array}$ & $\begin{array}{l}2 \\
4 \\
\end{array}$ & $\begin{array}{l}36 \\
36 \\
\end{array}$ & $\begin{array}{l}4 \\
5 \\
\end{array}$ & $\begin{array}{l}3 \\
5 \\
\end{array}$ & $\begin{array}{l}37 \\
35 \\
\end{array}$ \\
\hline \multirow{2}{*}{ SCOR } & $7^{\circ}$ & $\begin{array}{c}\text { fix } \\
\text { float }\end{array}$ & $\begin{array}{l}4 \\
4 \\
\end{array}$ & $\begin{array}{l}4 \\
5 \\
\end{array}$ & $\begin{array}{l}15 \\
15 \\
\end{array}$ & $\begin{array}{l}4 \\
6 \\
\end{array}$ & $\begin{array}{l}4 \\
1 \\
\end{array}$ & $\begin{array}{c}16 \\
9 \\
\end{array}$ & $\begin{array}{l}5 \\
5 \\
\end{array}$ & $\begin{array}{l}5 \\
6 \\
\end{array}$ & $\begin{array}{l}16 \\
18 \\
\end{array}$ \\
\hline & $25^{\circ}$ & $\begin{array}{l}\text { fix } \\
\text { float }\end{array}$ & $\begin{array}{l}4 \\
4\end{array}$ & $\begin{array}{l}3 \\
6\end{array}$ & $\begin{array}{c}10 \\
9\end{array}$ & $\begin{array}{l}4 \\
6\end{array}$ & $\begin{array}{l}3 \\
3\end{array}$ & $\begin{array}{c}12 \\
5\end{array}$ & $\begin{array}{l}5 \\
5\end{array}$ & $\begin{array}{l}4 \\
6 \\
\end{array}$ & $\begin{array}{l}17 \\
16\end{array}$ \\
\hline \multirow{2}{*}{ TIXI } & $7^{\circ}$ & $\begin{array}{l}\text { fix } \\
\text { float }\end{array}$ & $\begin{array}{l}1 \\
2\end{array}$ & $\begin{array}{l}1 \\
2\end{array}$ & $\begin{array}{l}3 \\
3 \\
\end{array}$ & $\begin{array}{l}1 \\
2\end{array}$ & $\begin{array}{l}1 \\
2\end{array}$ & $\begin{array}{l}4 \\
6 \\
\end{array}$ & $\begin{array}{l}3 \\
4\end{array}$ & $\begin{array}{l}2 \\
4 \\
\end{array}$ & $\begin{array}{l}11 \\
12 \\
\end{array}$ \\
\hline & $25^{\circ}$ & $\begin{array}{l}\text { fix } \\
\text { float }\end{array}$ & $\begin{array}{l}1 \\
2\end{array}$ & $\begin{array}{l}2 \\
2 \\
\end{array}$ & $\begin{array}{l}10 \\
10\end{array}$ & $\begin{array}{l}2 \\
2 \\
\end{array}$ & $\begin{array}{l}2 \\
3 \\
\end{array}$ & $\begin{array}{l}14 \\
14\end{array}$ & $\begin{array}{l}2 \\
4\end{array}$ & $\begin{array}{l}3 \\
5\end{array}$ & $\begin{array}{l}19 \\
24\end{array}$ \\
\hline \multirow{2}{*}{ ZAMB } & $7^{\circ}$ & $\begin{array}{c}\text { fix } \\
\text { float }\end{array}$ & $\begin{array}{l}2 \\
2 \\
\end{array}$ & $\begin{array}{l}2 \\
3 \\
\end{array}$ & $\begin{array}{l}4 \\
5 \\
\end{array}$ & $\begin{array}{l}2 \\
2 \\
\end{array}$ & $\begin{array}{l}2 \\
4 \\
\end{array}$ & $\begin{array}{l}5 \\
6 \\
\end{array}$ & $\begin{array}{l}3 \\
3 \\
\end{array}$ & $\begin{array}{l}3 \\
6 \\
\end{array}$ & $\begin{array}{c}8 \\
10 \\
\end{array}$ \\
\hline & $25^{\circ}$ & $\begin{array}{l}\text { fix } \\
\text { float }\end{array}$ & $\begin{array}{l}3 \\
3\end{array}$ & $\begin{array}{l}2 \\
4\end{array}$ & $\begin{array}{l}23 \\
24\end{array}$ & $\begin{array}{l}3 \\
3\end{array}$ & $\begin{array}{l}3 \\
6\end{array}$ & $\begin{array}{l}24 \\
26\end{array}$ & $\begin{array}{l}4 \\
4\end{array}$ & $\begin{array}{l}12 \\
12\end{array}$ & $\begin{array}{l}26 \\
31\end{array}$ \\
\hline
\end{tabular}

Table 4. RMS of stations used for PPP processing for 4-2-1 h (values are in $\mathrm{mm}$ )

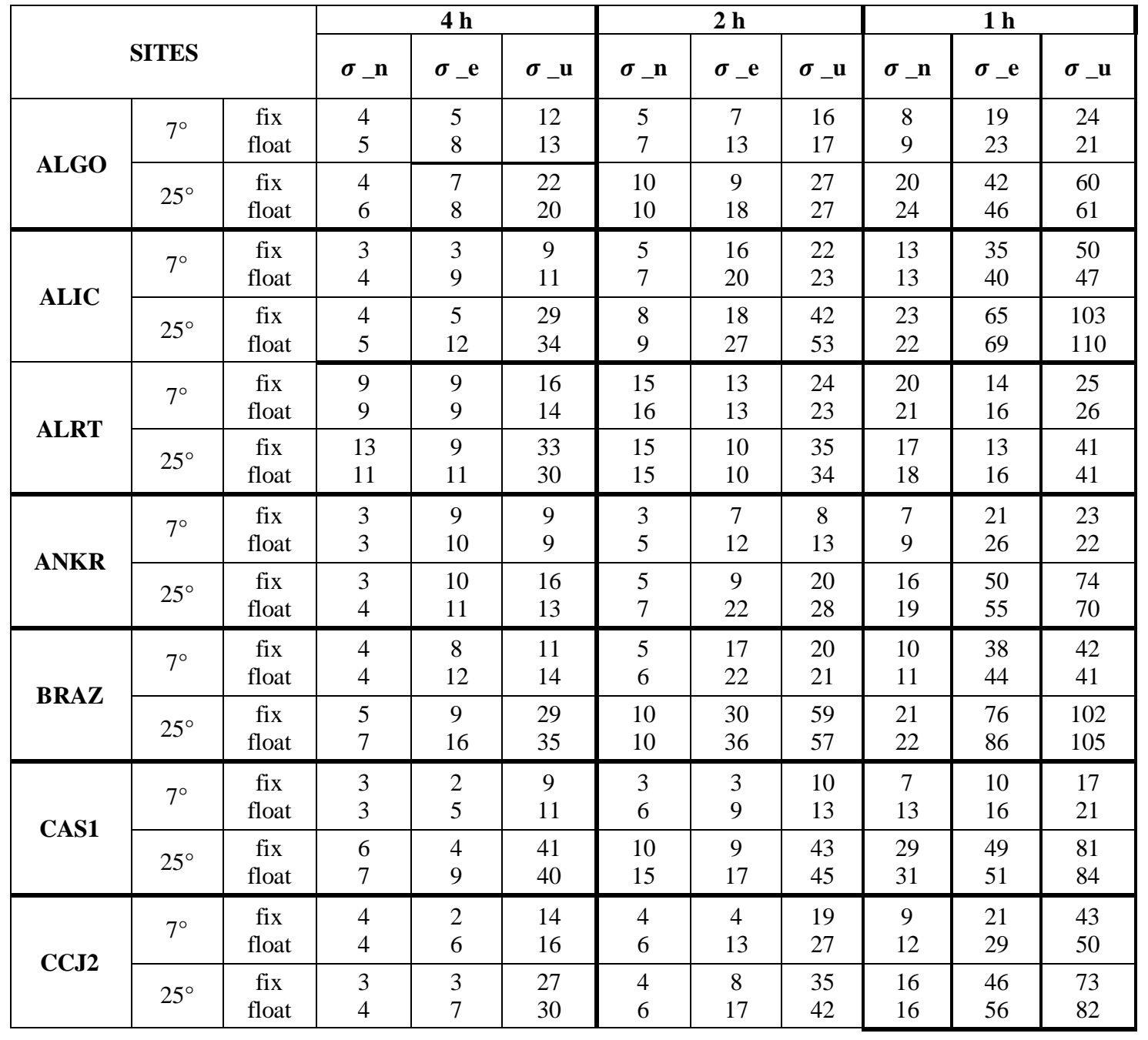


International Journal of Engineering and Geosciences (IJEG),

Vol; 5, Issue; 2, pp. 073-093, February, 2020,

\begin{tabular}{|c|c|c|c|c|c|c|c|c|c|c|c|}
\hline \multirow{2}{*}{ CKIS } & \multirow{2}{*}{$7^{\circ}$} & fix & 4 & 4 & 13 & 5 & 11 & 23 & 11 & 40 & 55 \\
& & float & 4 & 9 & 15 & 7 & 18 & 25 & 12 & 43 & 52 \\
\hline
\end{tabular}

Table 4 (Continued)

\begin{tabular}{|c|c|c|c|c|c|c|c|c|c|c|c|}
\hline & $25^{\circ}$ & $\begin{array}{l}\text { fix } \\
\text { float }\end{array}$ & $\begin{array}{l}6 \\
5\end{array}$ & $\begin{array}{c}5 \\
10\end{array}$ & $\begin{array}{l}20 \\
24\end{array}$ & $\begin{array}{l}8 \\
9\end{array}$ & $\begin{array}{l}14 \\
24\end{array}$ & $\begin{array}{l}35 \\
44\end{array}$ & $\begin{array}{l}19 \\
19\end{array}$ & $\begin{array}{l}57 \\
64\end{array}$ & $\begin{array}{l}84 \\
92\end{array}$ \\
\hline \multirow{2}{*}{ MAS1 } & $7^{\circ}$ & $\begin{array}{l}\text { fix } \\
\text { float }\end{array}$ & $\begin{array}{l}4 \\
4\end{array}$ & $\begin{array}{l}3 \\
7\end{array}$ & $\begin{array}{l}10 \\
13\end{array}$ & $\begin{array}{l}5 \\
6\end{array}$ & $\begin{array}{l}12 \\
17\end{array}$ & $\begin{array}{l}20 \\
22\end{array}$ & $\begin{array}{c}8 \\
18\end{array}$ & $\begin{array}{l}32 \\
54\end{array}$ & $\begin{array}{l}40 \\
81\end{array}$ \\
\hline & $25^{\circ}$ & $\begin{array}{c}\text { fix } \\
\text { float }\end{array}$ & $\begin{array}{l}5 \\
5\end{array}$ & $\begin{array}{c}5 \\
11\end{array}$ & $\begin{array}{l}18 \\
21 \\
\end{array}$ & $\begin{array}{l}7 \\
9 \\
\end{array}$ & $\begin{array}{l}17 \\
23 \\
\end{array}$ & $\begin{array}{l}35 \\
38 \\
\end{array}$ & $\begin{array}{l}17 \\
18 \\
\end{array}$ & $\begin{array}{l}51 \\
54 \\
\end{array}$ & $\begin{array}{l}72 \\
81 \\
\end{array}$ \\
\hline \multirow{2}{*}{ MAW1 } & $7^{\circ}$ & $\begin{array}{l}\text { fix } \\
\text { float }\end{array}$ & $\begin{array}{l}2 \\
3 \\
\end{array}$ & $\begin{array}{l}2 \\
4 \\
\end{array}$ & $\begin{array}{l}8 \\
8 \\
\end{array}$ & $\begin{array}{l}3 \\
5 \\
\end{array}$ & $\begin{array}{l}3 \\
8 \\
\end{array}$ & $\begin{array}{l}10 \\
11 \\
\end{array}$ & $\begin{array}{c}9 \\
23 \\
\end{array}$ & $\begin{array}{c}9 \\
37 \\
\end{array}$ & $\begin{array}{l}20 \\
50 \\
\end{array}$ \\
\hline & $25^{\circ}$ & $\begin{array}{c}\text { fix } \\
\text { float }\end{array}$ & $\begin{array}{l}5 \\
5\end{array}$ & $\begin{array}{l}3 \\
6 \\
\end{array}$ & $\begin{array}{l}14 \\
14\end{array}$ & $\begin{array}{l}7 \\
9 \\
\end{array}$ & $\begin{array}{c}5 \\
11 \\
\end{array}$ & $\begin{array}{l}21 \\
23 \\
\end{array}$ & $\begin{array}{l}22 \\
23\end{array}$ & $\begin{array}{l}35 \\
37 \\
\end{array}$ & $\begin{array}{l}50 \\
50\end{array}$ \\
\hline \multirow{2}{*}{ MCM4 } & $7^{\circ}$ & $\begin{array}{l}\text { fix } \\
\text { float }\end{array}$ & $\begin{array}{l}4 \\
5\end{array}$ & $\begin{array}{l}3 \\
5\end{array}$ & $\begin{array}{l}15 \\
17\end{array}$ & $\begin{array}{l}5 \\
8\end{array}$ & $\begin{array}{c}8 \\
10\end{array}$ & $\begin{array}{l}18 \\
22\end{array}$ & $\begin{array}{l}10 \\
14\end{array}$ & $\begin{array}{l}12 \\
15\end{array}$ & $\begin{array}{l}28 \\
31 \\
\end{array}$ \\
\hline & $25^{\circ}$ & $\begin{array}{c}\text { fix } \\
\text { float }\end{array}$ & $\begin{array}{l}6 \\
6\end{array}$ & $\begin{array}{l}5 \\
7\end{array}$ & $\begin{array}{l}29 \\
29\end{array}$ & $\begin{array}{c}10 \\
9\end{array}$ & $\begin{array}{c}8 \\
10\end{array}$ & $\begin{array}{l}34 \\
36\end{array}$ & $\begin{array}{l}19 \\
19\end{array}$ & $\begin{array}{l}20 \\
22\end{array}$ & $\begin{array}{l}57 \\
59\end{array}$ \\
\hline \multirow{2}{*}{ NOVM } & $7^{\circ}$ & $\begin{array}{l}\text { fix } \\
\text { float }\end{array}$ & $\begin{array}{l}4 \\
8\end{array}$ & $\begin{array}{c}2 \\
15\end{array}$ & $\begin{array}{l}28 \\
33\end{array}$ & $\begin{array}{c}8 \\
12\end{array}$ & $\begin{array}{l}16 \\
28\end{array}$ & $\begin{array}{l}34 \\
42\end{array}$ & $\begin{array}{l}17 \\
44\end{array}$ & $\begin{array}{l}29 \\
67\end{array}$ & $\begin{array}{c}54 \\
107\end{array}$ \\
\hline & $25^{\circ}$ & $\begin{array}{l}\text { fix } \\
\text { float }\end{array}$ & $\begin{array}{c}8 \\
11\end{array}$ & $\begin{array}{c}5 \\
10\end{array}$ & $\begin{array}{l}93 \\
90\end{array}$ & $\begin{array}{l}12 \\
19\end{array}$ & $\begin{array}{l}27 \\
34\end{array}$ & $\begin{array}{l}93 \\
84\end{array}$ & $\begin{array}{l}39 \\
39\end{array}$ & $\begin{array}{l}72 \\
74\end{array}$ & $\begin{array}{l}118 \\
106\end{array}$ \\
\hline \multirow{2}{*}{ NYA1 } & $7^{\circ}$ & $\begin{array}{c}\text { fix } \\
\text { float }\end{array}$ & $\begin{array}{l}2 \\
4 \\
\end{array}$ & $\begin{array}{l}3 \\
4 \\
\end{array}$ & $\begin{array}{l}7 \\
9 \\
\end{array}$ & $\begin{array}{l}3 \\
6 \\
\end{array}$ & $\begin{array}{l}3 \\
7 \\
\end{array}$ & $\begin{array}{l}11 \\
14 \\
\end{array}$ & $\begin{array}{c}6 \\
10 \\
\end{array}$ & $\begin{array}{c}6 \\
11 \\
\end{array}$ & $\begin{array}{l}18 \\
20 \\
\end{array}$ \\
\hline & $25^{\circ}$ & $\begin{array}{l}\text { fix } \\
\text { float }\end{array}$ & $\begin{array}{r}4 \\
6 \\
\end{array}$ & $\begin{array}{l}4 \\
5 \\
\end{array}$ & $\begin{array}{l}45 \\
41 \\
\end{array}$ & $\begin{array}{l}6 \\
8 \\
\end{array}$ & $\begin{array}{l}5 \\
8 \\
\end{array}$ & $\begin{array}{l}46 \\
48 \\
\end{array}$ & $\begin{array}{l}12 \\
14 \\
\end{array}$ & $\begin{array}{l}12 \\
15 \\
\end{array}$ & $\begin{array}{l}60 \\
62 \\
\end{array}$ \\
\hline \multirow{2}{*}{ ROTH } & $7^{\circ}$ & $\begin{array}{l}\text { fix } \\
\text { float }\end{array}$ & $\begin{array}{l}3 \\
3 \\
\end{array}$ & $\begin{array}{l}3 \\
5 \\
\end{array}$ & $\begin{array}{l}8 \\
9 \\
\end{array}$ & $\begin{array}{l}3 \\
6 \\
\end{array}$ & $\begin{array}{l}3 \\
8 \\
\end{array}$ & $\begin{array}{l}10 \\
11 \\
\end{array}$ & $\begin{array}{l}4 \\
9 \\
\end{array}$ & $\begin{array}{c}5 \\
14 \\
\end{array}$ & $\begin{array}{l}14 \\
17 \\
\end{array}$ \\
\hline & $25^{\circ}$ & $\begin{array}{l}\text { fix } \\
\text { float }\end{array}$ & $\begin{array}{l}5 \\
5 \\
\end{array}$ & $\begin{array}{l}4 \\
7\end{array}$ & $\begin{array}{l}39 \\
37\end{array}$ & $\begin{array}{l}7 \\
9 \\
\end{array}$ & $\begin{array}{c}4 \\
11 \\
\end{array}$ & $\begin{array}{l}41 \\
40 \\
\end{array}$ & $\begin{array}{l}14 \\
17\end{array}$ & $\begin{array}{l}22 \\
28 \\
\end{array}$ & $\begin{array}{l}53 \\
54 \\
\end{array}$ \\
\hline \multirow{2}{*}{ SCOR } & $7^{\circ}$ & $\begin{array}{l}\text { fix } \\
\text { float }\end{array}$ & $\begin{array}{l}5 \\
5 \\
\end{array}$ & $\begin{array}{l}5 \\
8 \\
\end{array}$ & $\begin{array}{l}16 \\
18 \\
\end{array}$ & $\begin{array}{l}5 \\
7 \\
\end{array}$ & $\begin{array}{c}6 \\
10 \\
\end{array}$ & $\begin{array}{l}19 \\
20 \\
\end{array}$ & $\begin{array}{l}12 \\
14 \\
\end{array}$ & $\begin{array}{l}13 \\
17 \\
\end{array}$ & $\begin{array}{l}27 \\
28 \\
\end{array}$ \\
\hline & $25^{\circ}$ & $\begin{array}{l}\text { fix } \\
\text { float }\end{array}$ & $\begin{array}{l}6 \\
6 \\
\end{array}$ & $\begin{array}{l}5 \\
9 \\
\end{array}$ & $\begin{array}{l}21 \\
18 \\
\end{array}$ & $\begin{array}{l}9 \\
9 \\
\end{array}$ & $\begin{array}{c}7 \\
12 \\
\end{array}$ & $\begin{array}{l}30 \\
26 \\
\end{array}$ & $\begin{array}{l}23 \\
23\end{array}$ & $\begin{array}{l}29 \\
31 \\
\end{array}$ & $\begin{array}{l}53 \\
45\end{array}$ \\
\hline \multirow{2}{*}{ TIXI } & $7^{\circ}$ & $\begin{array}{l}\text { fix } \\
\text { float }\end{array}$ & $\begin{array}{l}3 \\
4 \\
\end{array}$ & $\begin{array}{l}2 \\
6 \\
\end{array}$ & $\begin{array}{l}12 \\
15 \\
\end{array}$ & $\begin{array}{l}5 \\
8 \\
\end{array}$ & $\begin{array}{c}5 \\
10 \\
\end{array}$ & $\begin{array}{l}18 \\
20 \\
\end{array}$ & $\begin{array}{c}8 \\
15 \\
\end{array}$ & $\begin{array}{l}10 \\
18 \\
\end{array}$ & $\begin{array}{l}29 \\
37 \\
\end{array}$ \\
\hline & $25^{\circ}$ & $\begin{array}{l}\text { fix } \\
\text { float }\end{array}$ & $\begin{array}{l}4 \\
8\end{array}$ & $\begin{array}{l}5 \\
8 \\
\end{array}$ & $\begin{array}{l}23 \\
30\end{array}$ & $\begin{array}{c}8 \\
15\end{array}$ & $\begin{array}{c}9 \\
15\end{array}$ & $\begin{array}{l}42 \\
43\end{array}$ & $\begin{array}{l}28 \\
30\end{array}$ & $\begin{array}{l}41 \\
47\end{array}$ & $\begin{array}{l}82 \\
89\end{array}$ \\
\hline \multirow{2}{*}{ ZAMB } & $7^{\circ}$ & $\begin{array}{l}\text { fix } \\
\text { float }\end{array}$ & $\begin{array}{l}3 \\
3 \\
\end{array}$ & $\begin{array}{l}7 \\
9 \\
\end{array}$ & $\begin{array}{l}11 \\
13 \\
\end{array}$ & $\begin{array}{l}6 \\
7 \\
\end{array}$ & $\begin{array}{l}12 \\
18 \\
\end{array}$ & $\begin{array}{l}28 \\
23\end{array}$ & $\begin{array}{l}12 \\
13 \\
\end{array}$ & $\begin{array}{l}31 \\
37 \\
\end{array}$ & $\begin{array}{l}44 \\
45 \\
\end{array}$ \\
\hline & $25^{\circ}$ & $\begin{array}{l}\text { fix } \\
\text { float }\end{array}$ & $\begin{array}{l}4 \\
5\end{array}$ & $\begin{array}{l}12 \\
15\end{array}$ & $\begin{array}{l}30 \\
33\end{array}$ & $\begin{array}{l}11 \\
11\end{array}$ & $\begin{array}{l}19 \\
26\end{array}$ & $\begin{array}{l}43 \\
48\end{array}$ & $\begin{array}{l}19 \\
23\end{array}$ & $\begin{array}{l}54 \\
58\end{array}$ & $\begin{array}{l}85 \\
87\end{array}$ \\
\hline
\end{tabular}



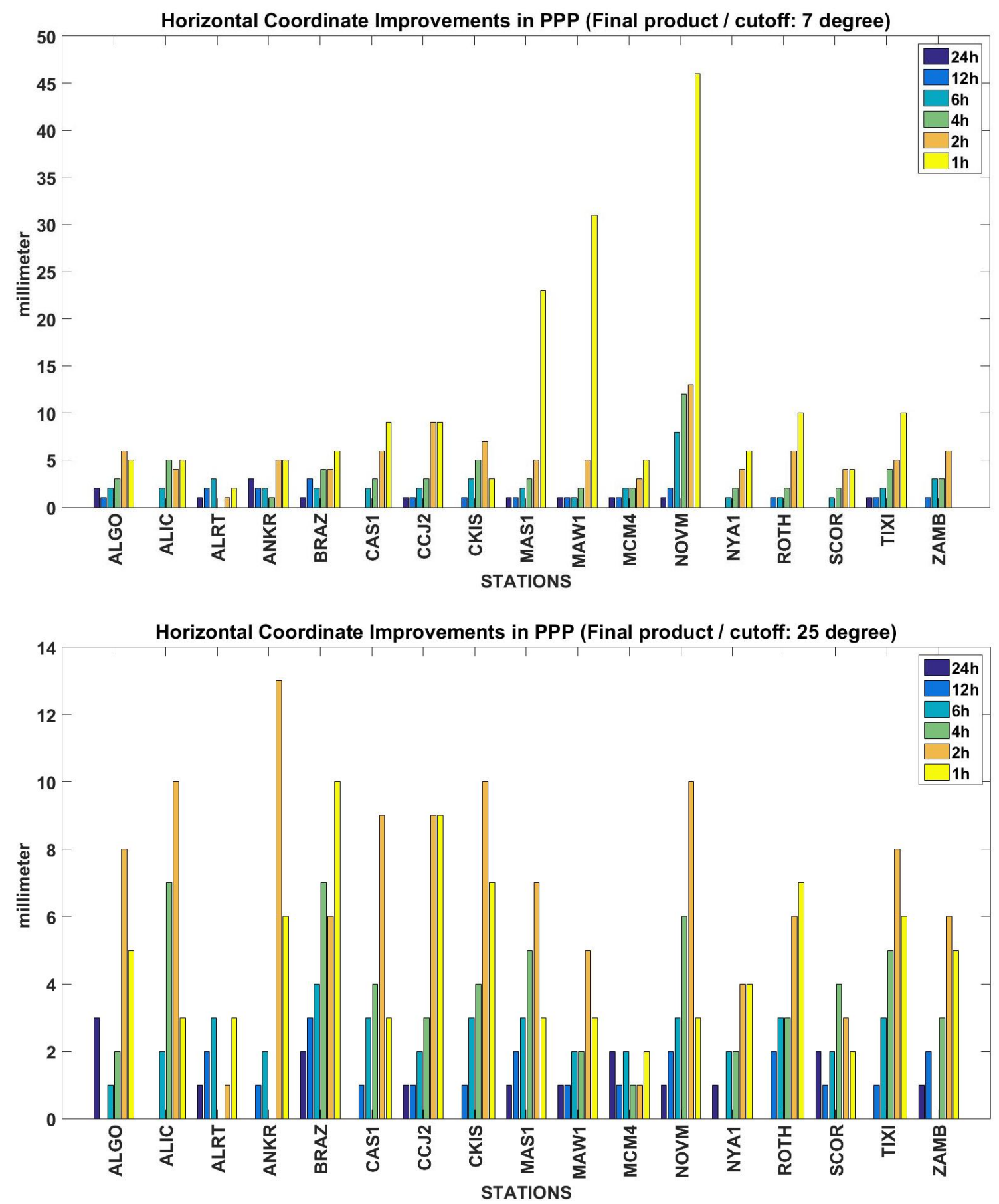

Figure 2. Horizontal coordinate improvements for PPP using final products with $7^{\circ} / 25^{\circ}$ cut-off angle 

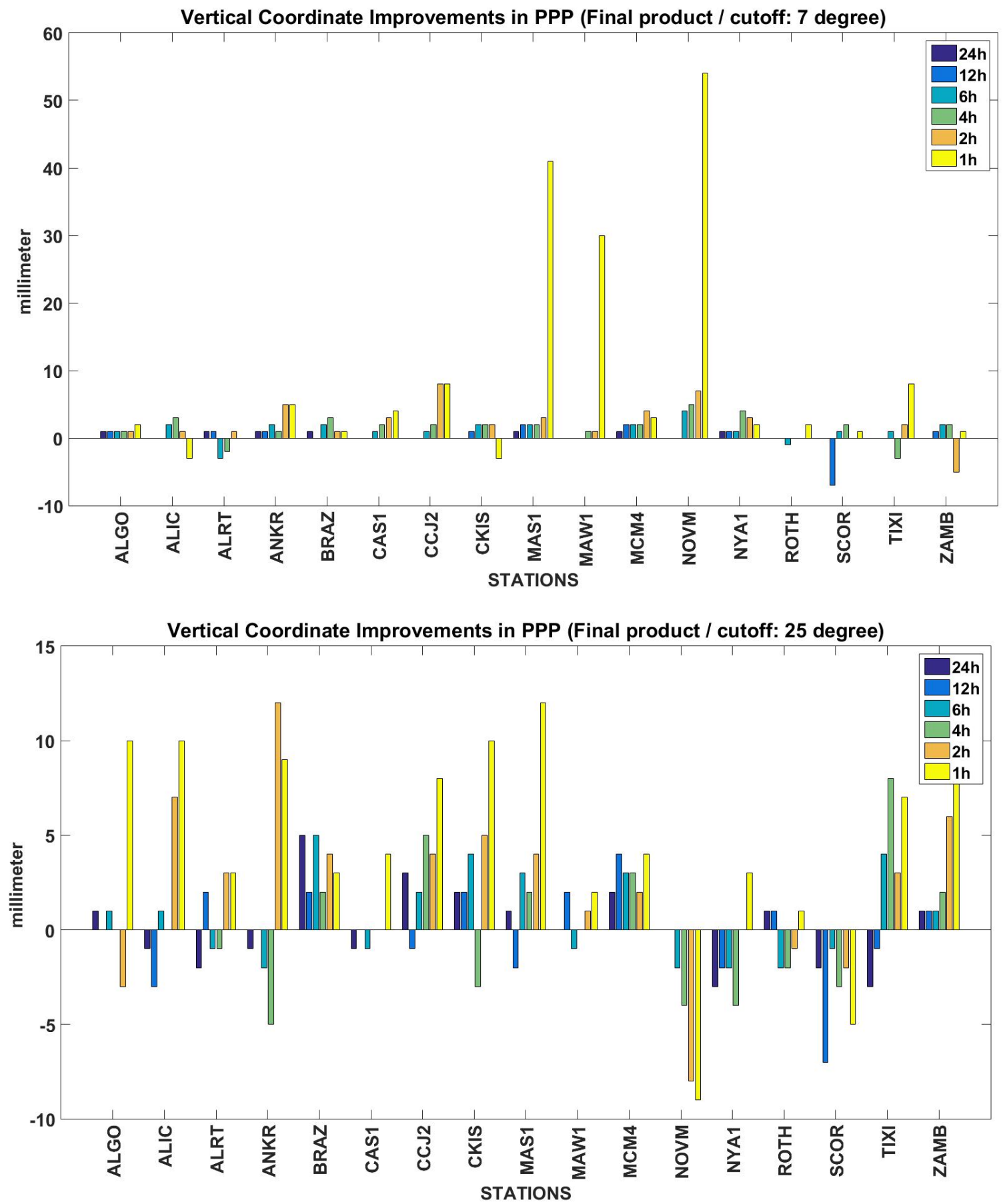

Figure 3. Vertical coordinate improvements for PPP using finals product with $7^{\circ} / 25^{\circ}$ cut-off angle 

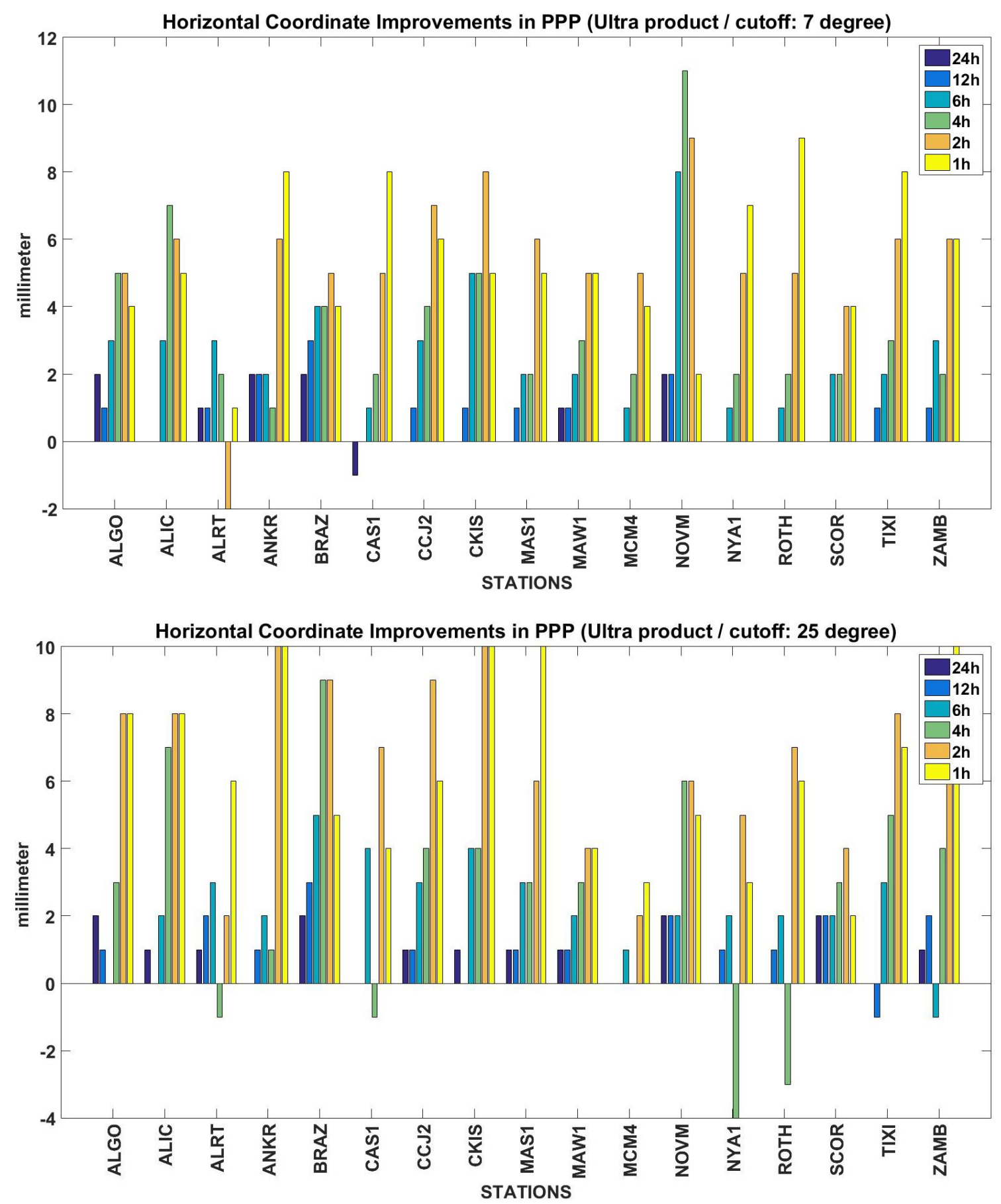

Figure 4. Horizontal coordinate improvements for PPP using ultra products with $7^{\circ} / 25^{\circ}$ cut-off angle 

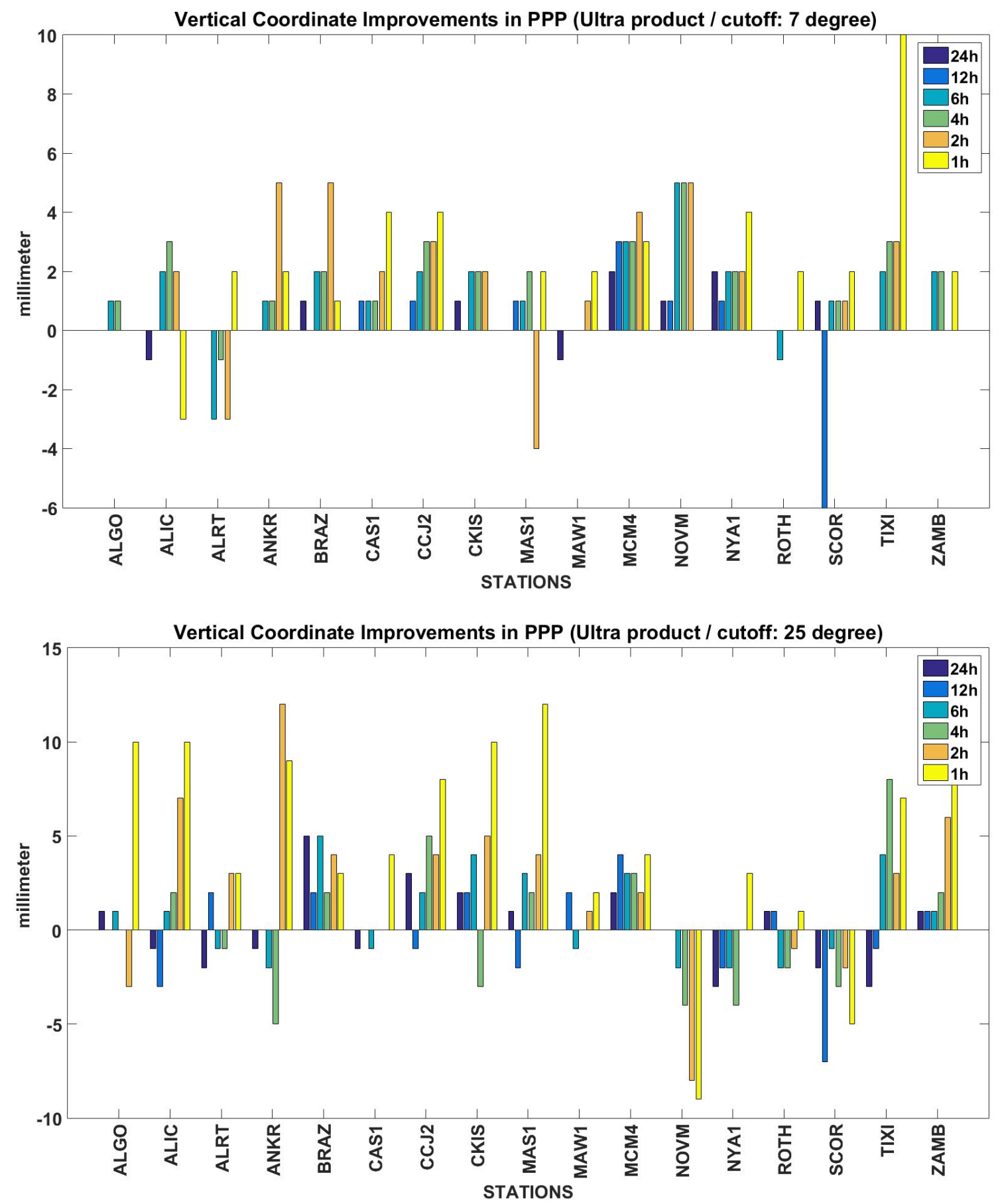

Figure 5. Vertical coordinate improvements for PPP using ultra product with $7^{\circ} / 25^{\circ}$ cut-off angle 
As can be seen from the PPP results, AR impacts on the estimated coordinates depended on the observation session, satellite geometry, and orbit/satellite product. As was expected, AR impact increased as the observation session length decreased. The results also showed that accuracy degradation of AR compared to the float solutions was generally observed with $25^{\circ}$ elevation cut-off angle due to the unstable AR under the poor satellite geometry. The results also revealed that final and ultra-rapid products affected the improvement of PPP-AR on the coordinates for each observation session but no trend was found with regards to the magnitude and direction of the AR impact on the coordinates.

Table 5. RMS of stations used for relative processing for 24-12-6 h (values are in $\mathrm{mm}$ )

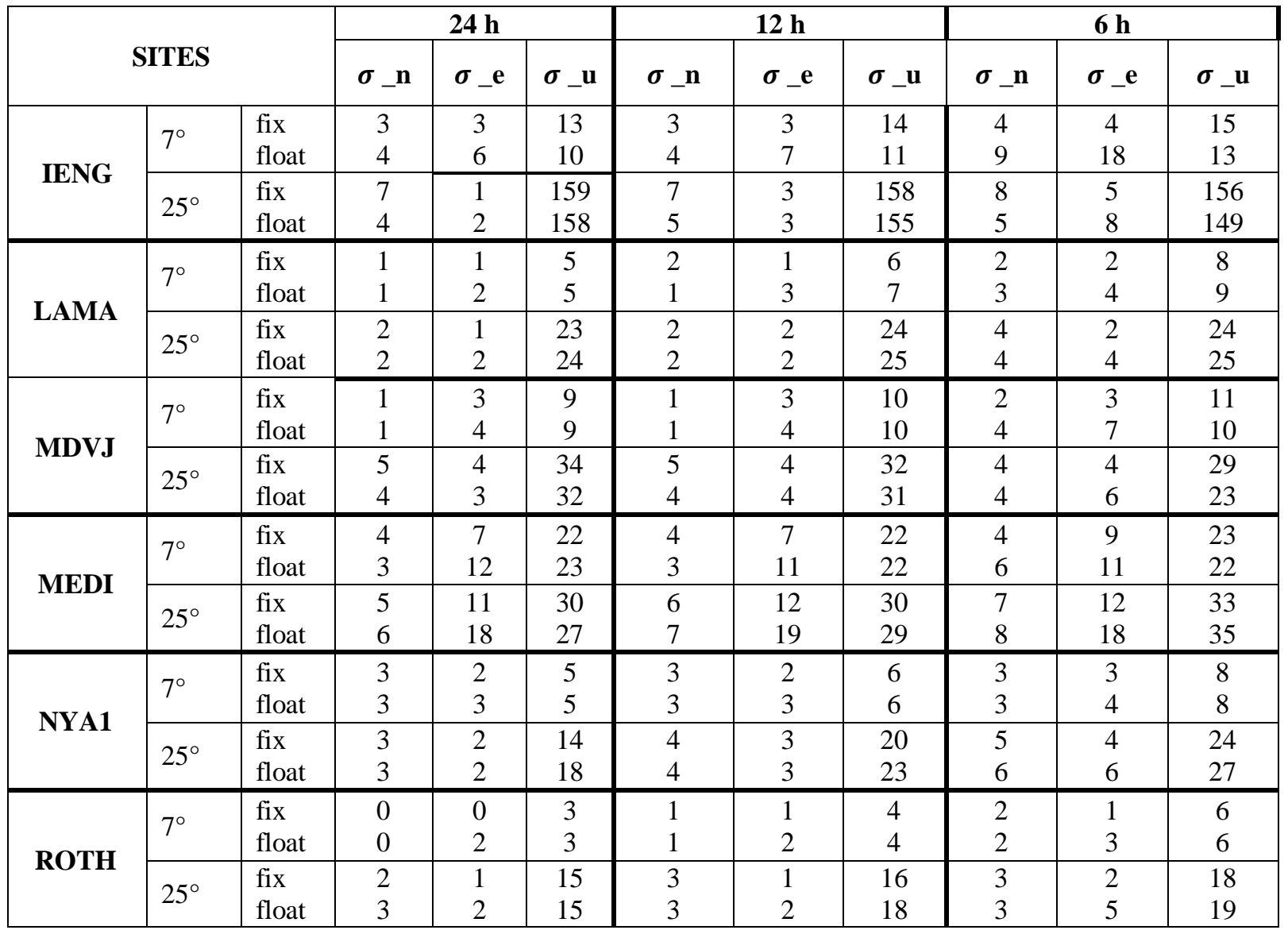


International Journal of Engineering and Geosciences (IJEG),

Vol; 5, Issue; 2, pp. 073-093, February, 2020,

Table 6. Statistics of stations used for relative processing for 4-2-1h (values are in $\mathrm{mm}$ )

\begin{tabular}{|c|c|c|c|c|c|c|c|c|c|c|c|}
\hline \multirow{2}{*}{\multicolumn{3}{|c|}{ SITES }} & \multicolumn{3}{|c|}{$4 \mathrm{~h}$} & \multicolumn{3}{|c|}{$2 \mathrm{~h}$} & \multicolumn{3}{|c|}{$1 \mathrm{~h}$} \\
\hline & & & $\begin{array}{ll}\boldsymbol{\sigma} & \mathbf{n}\end{array}$ & $\sigma_{\text {_ }} \mathbf{e}$ & $\sigma_{\quad} \mathbf{u}$ & $\begin{array}{ll}\boldsymbol{\sigma} & \mathbf{n}\end{array}$ & $\sigma_{\boldsymbol{\sigma}} \mathbf{e}$ & $\sigma_{\text {o }} \mathbf{u}$ & $\boldsymbol{\sigma} \quad \mathbf{n}$ & $\begin{array}{l}\boldsymbol{\sigma} \\
\mathbf{e}\end{array}$ & $\boldsymbol{\sigma} \mathbf{u}$ \\
\hline \multirow{4}{*}{ IENG } & 70 & fix & 6 & 7 & 18 & 18 & 43 & 43 & 40 & 82 & 94 \\
\hline & T & float & 11 & 22 & 21 & 23 & 51 & 52 & 43 & 83 & 97 \\
\hline & 250 & fix & 8 & 6 & 157 & 16 & 45 & 152 & 57 & 113 & 157 \\
\hline & 25 & float & 8 & 11 & 149 & 20 & 50 & 141 & 58 & 116 & 152 \\
\hline \multirow{4}{*}{ LAMA } & 70 & fix & 2 & 2 & 9 & 3 & 4 & 10 & 11 & 28 & 26 \\
\hline & T & float & 3 & 5 & 10 & 6 & 13 & 15 & 14 & 33 & 29 \\
\hline & 250 & fix & 4 & 2 & 27 & 8 & 14 & 35 & 27 & 64 & 67 \\
\hline & 25 & float & 6 & 6 & 27 & 11 & 21 & 35 & 29 & 68 & 71 \\
\hline \multirow{4}{*}{ MDVJ } & 70 & fix & 2 & 3 & 13 & 3 & 6 & 15 & 11 & 26 & 33 \\
\hline & p & float & 5 & 7 & 12 & 6 & 13 & 15 & 15 & 32 & 36 \\
\hline & $25^{\circ}$ & fix & 5 & 5 & 33 & 6 & 12 & 35 & 51 & 88 & 99 \\
\hline & 25 & float & 5 & 7 & 28 & 9 & 19 & 36 & 52 & 90 & 102 \\
\hline \multirow{4}{*}{ MEDI } & 70 & fix & 6 & 10 & 26 & 10 & 24 & 36 & 31 & 81 & 85 \\
\hline & T & float & 6 & 16 & 30 & 13 & 31 & 42 & 32 & 83 & 87 \\
\hline & $25^{\circ}$ & fix & 8 & 15 & 39 & 16 & 41 & 69 & 53 & 116 & 142 \\
\hline & 25 & float & 10 & 21 & 46 & 19 & 50 & 79 & 54 & 121 & 141 \\
\hline \multirow{4}{*}{ NYA1 } & 70 & fix & 9 & 9 & 16 & 10 & 6 & 20 & 14 & 11 & 27 \\
\hline & p & float & 10 & 10 & 17 & 11 & 8 & 21 & 18 & 15 & 30 \\
\hline & $25^{\circ}$ & fix & 7 & 5 & 35 & 10 & 16 & 46 & 35 & 26 & 98 \\
\hline & $25^{\circ}$ & float & 6 & 6 & 36 & 12 & 17 & 50 & 38 & 27 & 101 \\
\hline \multirow{4}{*}{ ROTH } & 70 & & 2 & 1 & 7 & 2 & 1 & 6 & 2 & 1 & 7 \\
\hline & r & float & 3 & 4 & 7 & 2 & 3 & 6 & 3 & 4 & 7 \\
\hline & $25^{\circ}$ & fix & 4 & 2 & 19 & 3 & 2 & 18 & 4 & 2 & 19 \\
\hline & 25 & float & 4 & 7 & 20 & 3 & 5 & 19 & 4 & 7 & 20 \\
\hline
\end{tabular}



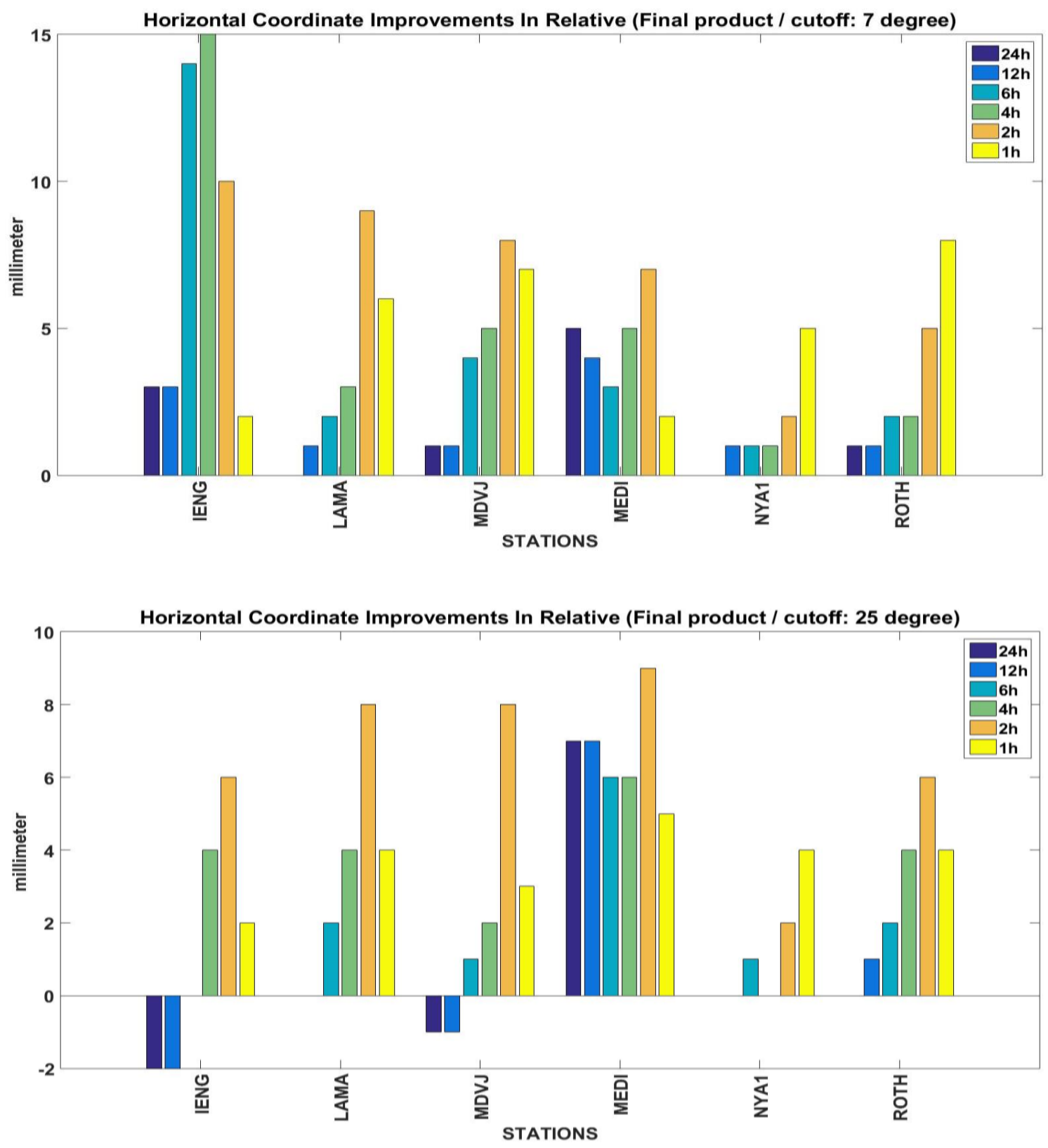

Figure 6. Horizontal coordinates improvements for relative using final product with $7 \% 25^{\circ}$ cut-off angle 

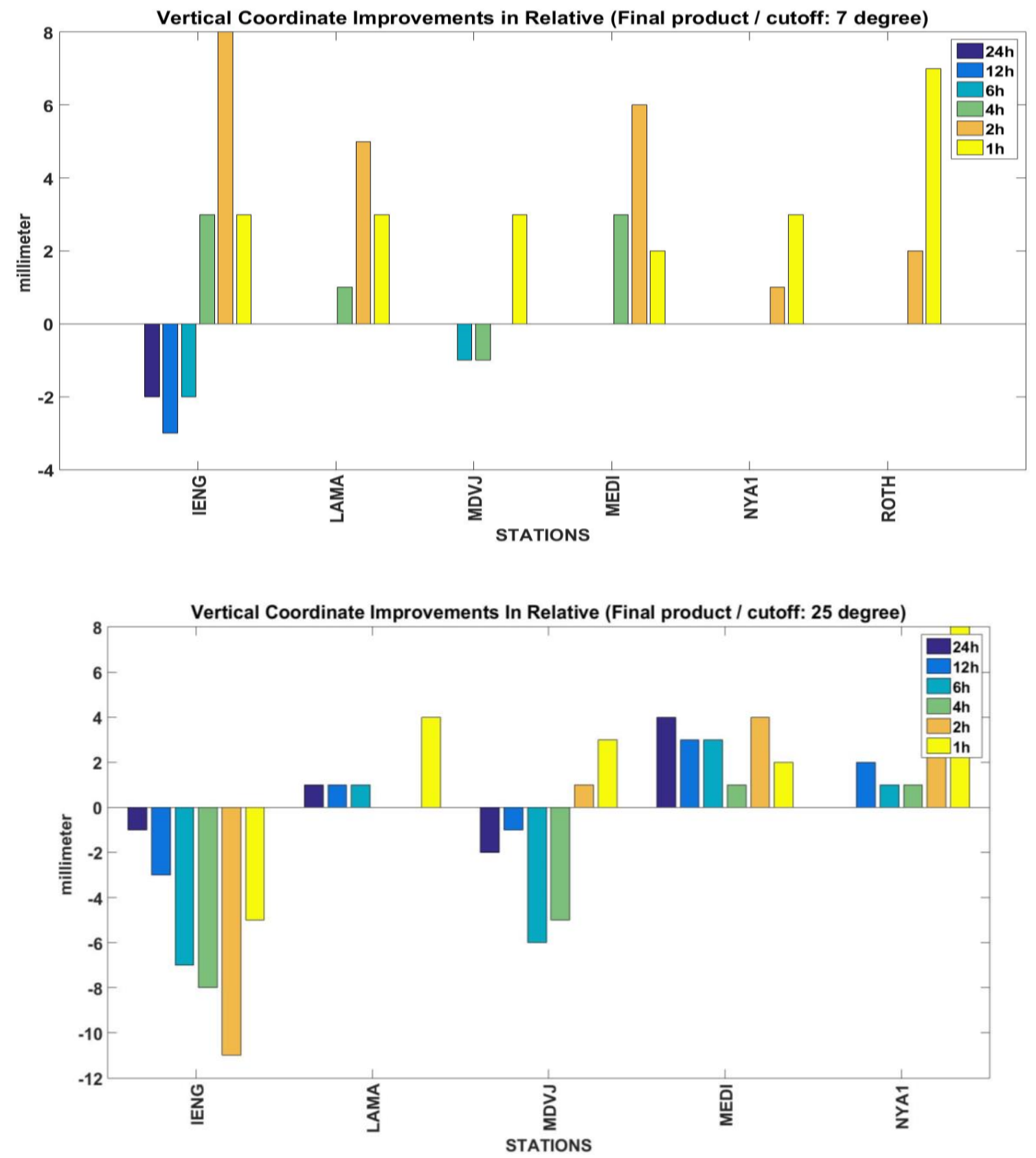

Figure 7. Vertical coordinates improvements for relative using final product with $7 \% 25^{\circ}$ cut-off angle 
Relative processing results show that the AR impact on the estimated coordinates did not depend on the baseline length. The accuracy degradation of AR compared to float solutions was generally observed with $25^{\circ}$ elevation cut-off angle as in the PPP results. Due to the nearly identical results (sub-mm difference) between the final and ultrarapid products, AR improvement of ultra-rapid products was not given for the relative processing results. The maximum difference was observed for the longest baseline: a $0.5 \mathrm{~mm}$ change in the horizontal and a $0.9 \mathrm{~mm}$ change in the vertical. The main reason behind this is that satellite clock bias is removed using double differences, whereas in PPP, it directly lumps to the station coordinates. Orbit differences between the products were relatively small compared to the satellite clock bias. Table 7 shows the average RMSE values of the radial, along-track and crosstrack components of all GPS satellites between the final and ultra-rapid products for 31-day period in January 2018.

Table 7. Average RMSE values of radial (R), along-track (A) and cross-track (C) components of all GPS satellites during the observation period (units: $\mathrm{mm}$ )

\begin{tabular}{|c|c|c|}
\hline \multicolumn{3}{|c|}{ FINAL - ULTRA-RAPID } \\
\hline $\mathbf{R}$ & $\mathbf{A}$ & $\mathbf{C}$ \\
\hline 7 & 12 & 11 \\
\hline
\end{tabular}

The relationship between observation session and AR impact on the coordinates in relative processing was similar to that in PPP, but the magnitudes of improvement were much higher in PPP. Maximum improvement and degradation in coordinates of AR in horizontal and vertical components were 54/12 mm, -9/-4 mm and 15/10 mm, and $-2 /-11 \mathrm{~mm}$ for PPP and relative positioning, respectively. The AR improvement of each technique in the horizontal component was much bigger than the improvement in the vertical component for most of the stations.

Fixing to the wrong integer ambiguities was investigated for each technique and split into two categories. The wrong fix in the first category is assumed if any fixed solution is within the outliers, but its float solution is not an outlier. The wrong fix in the second category is assumed if any two-dimensional error of the fix solution is $20 \mathrm{~mm}$ higher than its error in the float solution. Since the impact of AR is highly correlated with the twodimensional, this assumption was applied. A $20 \mathrm{~mm}$ threshold was taken as an arbitrary value to exclude small biases between the fix and float solutions. Tables $8,-9,-10$ and -11 show the total number of wrong fix and their AR success rate for PPP and relative positioning respectively using the final product. Due to the fact that most of the wrong fixes are accumulated in $1 \mathrm{~h}$, the other sessions were omitted to save space in this manuscript. Some float solutions are also within the outliers except its fixed solutions. These float solutions within outliers are also shown in Table 8-10. The number of float solution outliers is significantly lower than the number of fixed solution outliers for PPP, but in relative positioning, the numbers of fixed and float outliers are similar except for IENG and MEDI stations. In these stations, the number of fixed solution outliers are higher than theirs float solution outliers.

The fixing rate is defined as the ratio of the ambiguities used over all the ambiguities, and is expressed as follows:

$F=\frac{N_{\text {used }_{\text {ambiguities }}}}{N_{\text {all }} \text { ambiguities }} * \mathbf{1 0 0} \%$

where $\mathrm{F}$ denotes the ambiguity fixing rate, $\boldsymbol{N}_{\text {used }_{\text {ambiguities }}}$ refers to the number of fixed ambiguities that are used for the coordinate estimations after the validation test, and $\boldsymbol{N}_{\text {all }_{\text {ambiguities }}}$ is the number of all ambiguities that have been found. 
International Journal of Engineering and Geosciences (IJEG),

Vol; 5, Issue; 2, pp. 073-093, February, 2020,

Table 8. Wrong fix within the first category for PPP

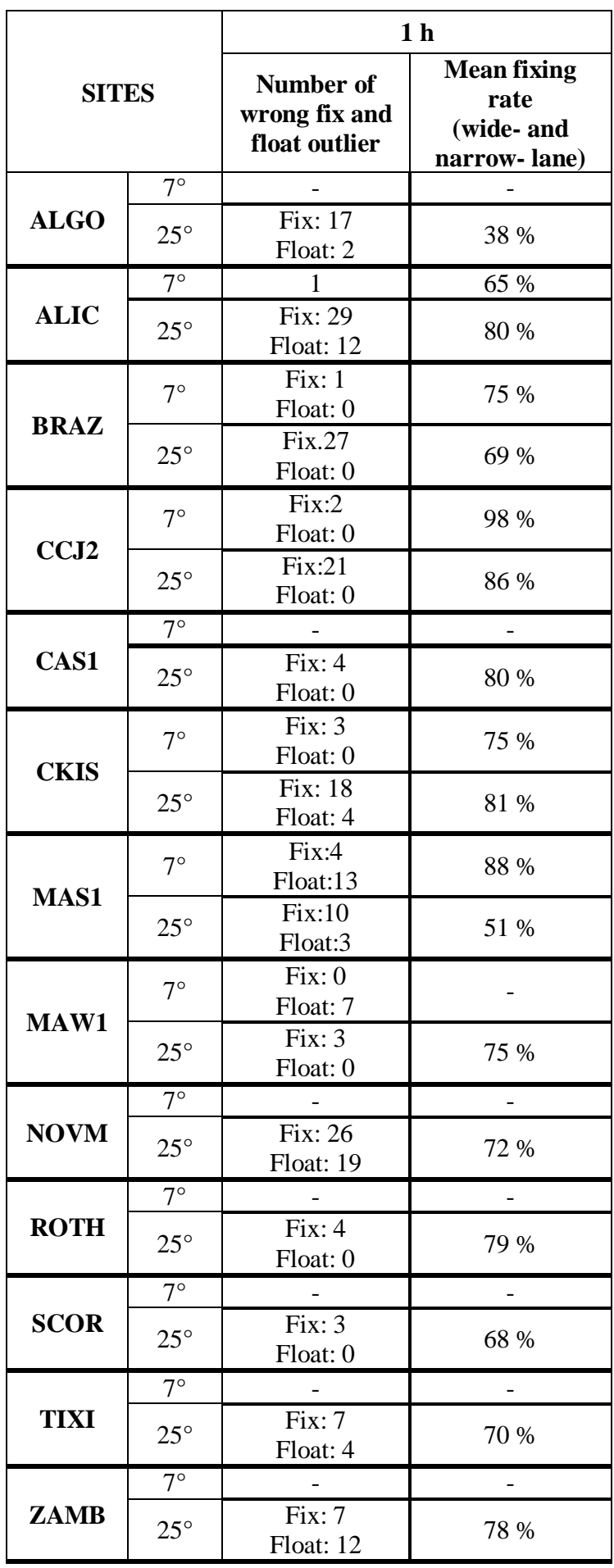

Table 9. Wrong fix within the second category for PPP

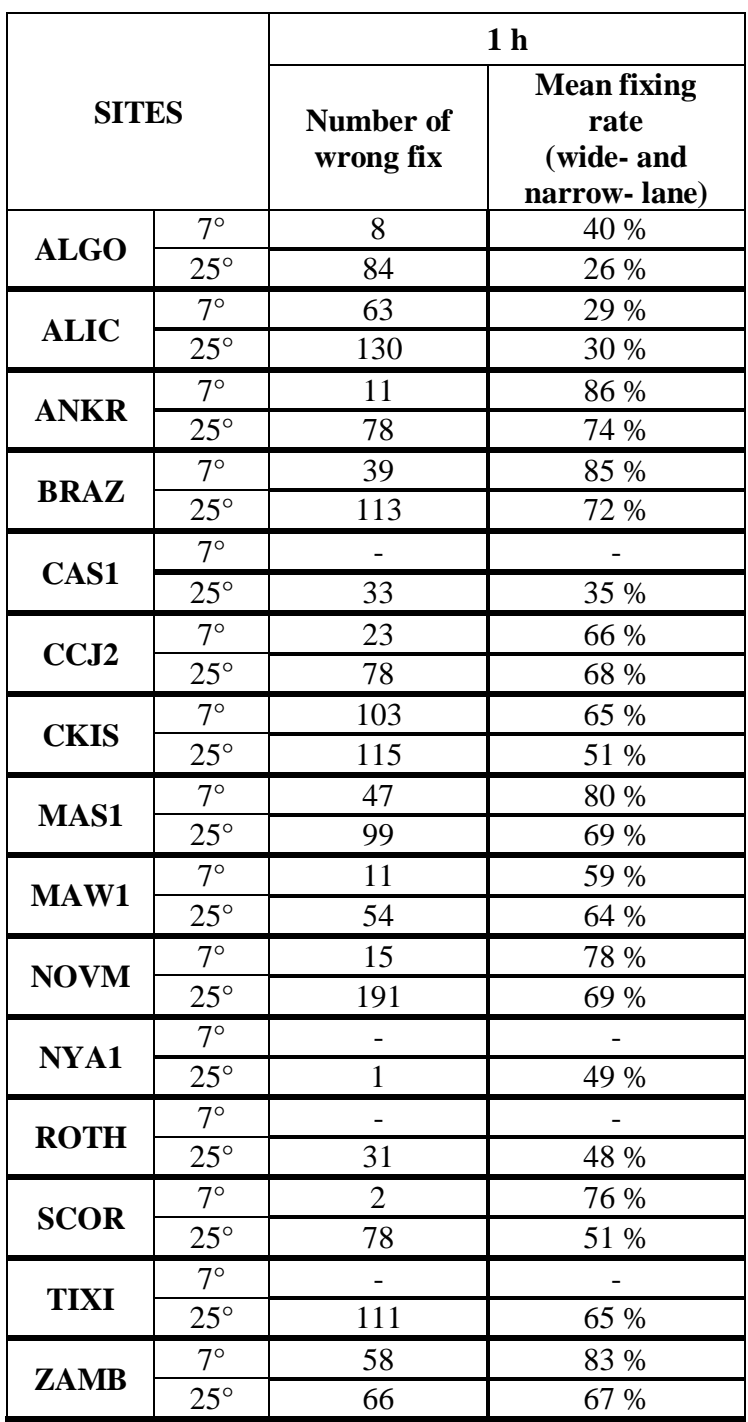


Table 10. Wrong fix within the first category for relative positioning

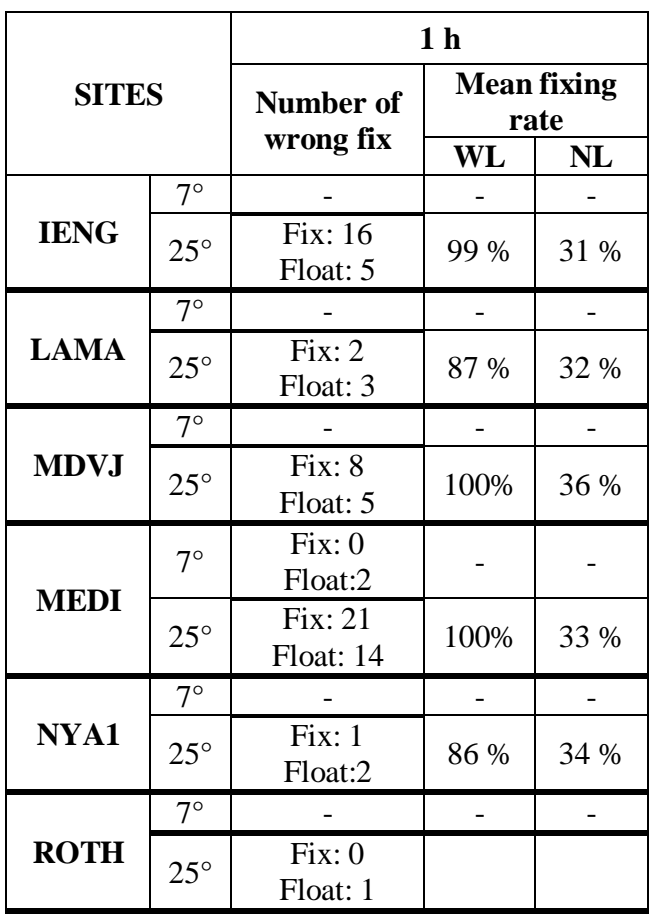

Wide-lane and narrow-lane fixing rates of outliers were given separately for relative positioning, but due to the software restrictions they were given a single mean value (wide and narrow-lane ambiguities) for PPP. The mean wide-lane and narrow-lane fixing rates of $1 \mathrm{~h}$ nonoutlier solutions using $7^{\circ}$ and $25^{\circ}$ cut-off angles were $95 \% / 59 \%$ and $99 \% / 58 \%$ for relative positioning, respectively. It is observed that the narrow-lane ambiguity fixing rate was significantly lower than the wide-lane ambiguity fixing due to its narrower wavelength. The mean wide and narrow-lane ambiguities fixing rates of $1 \mathrm{~h}$ non-outlier solutions using $7^{\circ}$ and $25^{\circ}$ cut-off angles were $79 \%$ and $70 \%$ for PPP respectively. Wrong fix results show that fixing to the wrong integer ambiguities occurred more frequently for the $25^{\circ}$ cut-off angle compared to the $7^{\circ}$ cut-off angle for each technique. This situation can be explained by the fact that arch length of the satellites is significantly smaller for the $25^{\circ}$ cut-off angle compared to the $7^{\circ}$ cut-off angle, and this leads to unreliable ambiguity resolution for each satellite.
Table 11. Wrong fix within the second category for relative positioning

\begin{tabular}{|c|c|c|c|c|}
\hline \multicolumn{2}{|c|}{ SITES } & \multicolumn{3}{c|}{1 h } \\
\cline { 3 - 5 } \multicolumn{2}{|c|}{$\begin{array}{c}\text { Number } \\
\text { of wrong } \\
\text { fix }\end{array}$} & \multicolumn{2}{c|}{ Mean fixing rate } \\
\cline { 3 - 5 } & & WL & NL \\
\hline \multirow{2}{*}{ IENG } & $7^{\circ}$ & 45 & $98 \%$ & $34 \%$ \\
\cline { 2 - 5 } & $25^{\circ}$ & 210 & $99 \%$ & $18 \%$ \\
\hline \multirow{2}{*}{ LAMA } & $7^{\circ}$ & 12 & $98 \%$ & $24 \%$ \\
\cline { 2 - 5 } & $25^{\circ}$ & 118 & $95 \%$ & $46 \%$ \\
\hline \multirow{2}{*}{ MDVJ } & $7^{\circ}$ & 23 & $100 \%$ & $50 \%$ \\
\cline { 2 - 5 } & $25^{\circ}$ & 208 & $100 \%$ & $39 \%$ \\
\hline \multirow{2}{*}{ MEDI } & $7^{\circ}$ & 114 & $98 \%$ & $22 \%$ \\
\cline { 2 - 5 } & $25^{\circ}$ & 209 & $100 \%$ & $34 \%$ \\
\hline \multirow{2}{*}{ NYA1 } & $7^{\circ}$ & 5 & $89 \%$ & $28 \%$ \\
\cline { 2 - 5 } & $25^{\circ}$ & 87 & $86 \%$ & $77 \%$ \\
\hline \multirow{2}{*}{ ROTH } & $7^{\circ}$ & 4 & $100 \%$ & $57 \%$ \\
\cline { 2 - 5 } & $25^{\circ}$ & 57 & $100 \%$ & $55 \%$ \\
\hline
\end{tabular}

\section{CONCLUSION}

Ambiguity resolution impacts on estimated coordinates for static PPP and relative positioning techniques are presented in this paper. Seventeen globally distributed stations and six baselines with varying lengths were chosen for conducting PPP and relative positioning respectively for a 31-day period in January 2018. Daily RINEX files of the stations were subdivided into mutually non-overlapping sessions as 24-12-6-4-2-1 h subsets. Ambiguity resolution impacts on the estimated coordinates were tested using $7^{\circ}$ and $25^{\circ}$ cut-off angles and final/ultra-rapid orbit and clock products. The results can be summarized as follows:

Ambiguity resolution impacts on the estimated coordinates increases as observation time decreases for PPP and relative positioning techniques respectively.

Using orbit/satellite products with differences in latency affects AR impacts on the estimated coordinates for PPP, especially for short observation sessions. However, it does not significantly affect the estimated coordinates for relative positioning due removal of the satellite clock bias in double difference. The biggest difference found between the products was $46 \mathrm{~mm}$ (2D) and $54 \mathrm{~mm}$ (up) in NOVM station for PPP processing.

The results reveal that accuracy degradation of $A R$ compared to the float solutions on the estimated coordinates are generally seen for up component when using the $25^{\circ}$ cut-off angle for PPP and relative positioning. Maximum positive and negative ambiguity resolution improvements in horizontal and vertical components were $54 \mathrm{~mm}\left(1 \mathrm{~h}, 7^{\circ}\right) / 12 \mathrm{~mm}\left(1 \mathrm{~h}, 25^{\circ}\right),-4$ $\mathrm{mm}\left(4 \mathrm{~h}, 25^{\circ}\right) /-9 \mathrm{~mm}\left(1 \mathrm{~h}, 25^{\circ}\right)$ and $15 \mathrm{~mm}\left(4 \mathrm{~h}, 7^{\circ}\right) / 10$ $\mathrm{mm}\left(2 \mathrm{~h}, 25^{\circ}\right),-2 \mathrm{~mm}\left(12 \mathrm{~h}, 25^{\circ}\right) /-11 \mathrm{~mm}\left(2 \mathrm{~h}, 25^{\circ}\right)$ for PPP and relative positioning respectively.

Fixing to the wrong integer ambiguities was investigated in two categories; first category was applied as when any fixed solution is within the outliers (errors greater than $30 \mathrm{~cm}$ ), but its float solution is not an outlier, and the second category was applied as when the fixed solution which its two-dimensional error was $20 \mathrm{~mm}$ higher than the error of the float solution. In accordance with the results, fixing to the wrong integer ambiguities 
are accumulated generally in $1 \mathrm{~h}$ solutions for each technique. The highest amount of fix outliers in the second category were found $26 \%$ and $29 \%$ of all data sets for PPP and relative positioning techniques with $25^{\circ}$ cutoff angle.

The results also reveal that fixing to the wrong integer ambiguities occurred more frequently for the $25^{\circ}$ cut-off angle compared to the $7^{\circ}$ cut-off angle for each technique due to the satellite arch length being inadequate to resolve ambiguities reliably. In accordance with the outlier results of the first category, fix outliers of PPP are $36 \%$ and $75 \%$ of all fix and float outliers using 7 and 25-degree cutoff angle respectively. Fix outliers of relative positioning are $0 \%$ and $60 \%$ of all fix and float outliers using 7 and 25 degree cutoff angle respectively.

If PPP or relative technique need to be applied with a short observation period (for example, $1 \mathrm{~h}$ ) under the obstructed conditions, the float solution can be chosen for a higher likelihood of avoiding the wrong fix solution. In terms of using AR under the poor satellite geometry with short observation time, partial ambiguity fixing or changing the AR validation might be conducted to avoid the wrong fix solution.

No direct relationship was observed between the baseline length and the coordinates' improvement of AR compared to the float solution.

\section{ACKNOWLEDGEMENTS}

The authors would like to express their gratitude to NASA Jet Propulsion Laboratory (JPL) and Massachusetts Institute of Technology (MIT) for providing GIPSY/OASIS and GAMIT/GLOBK scientific software.

\section{REFERENCES}

Alcay, S., Ogutcu, S., Kalayci, I and Yigit, C.O. (2019). Displacement monitoring performance of relative positioning and Precise Point Positioning (PPP) methods using simulation apparatus, Advances in Space Research $63,5,1697-1707$.

Bar - Sever, Y. E., Kroger, P. M., and Borjesson, J. A. (1998). Estimating horizontal gradients of tropospheric path delay with a single GPS receiver. Journal of Geophysical Research: Solid Earth, 103(B3), 5019-5035.

Blewitt G., Young LE., and Meehan TH. (1989). Subcentimeter baselines within seconds using ROGUE receivers: introducing the rapid static survey (RSS) method. In: International association of geodesy conference, Edinburgh, August, 1989.

Bezcioglu M., Yigit, O. C and El-Mowafy, A. (2019). Kinematic PPP-AR in Antarctic: Comparing Methods for Precise Positioning. Sea Technology.

Bertiger, W., Desai, S. D., Haines, B., Harvey, N., Moore, A. W., Owen, S and Weiss, J. P. (2010). Single receiver phase ambiguity resolution with GPS data. Journal of Geodesy, 84(5), 327-337.

Brach, M and Zasada, M. (2014). The effect of mounting height on GNSS receiver positioning accuracy in forest conditions. Croatian Journal of Forest Engineering: Journal for Theory and Application of Forestry Engineering, 35(2), 245-253.

Chen, H., Xiao, Y., Jiang, W., Zhou, X and Liu, H. (2017). An improved method for multi-GNSS baseline processing using single difference. Advances in Space Research.

Choy, S., Bisnath, S and Rizos, C. (2017). Uncovering common misconceptions in GNSS Precise Point Positioning and its future prospect. GPS solutions, 21(1), 13-22.

Gandolfi, S., Tavasci, L and Poluzzi, L. (2017). Study on GPS-PPP precision for short observation sessions. GPS Solutions, 21(3), 887-896.

Ge, M., Gendt, G., Rothacher, M. A., Shi, C and Liu, J. (2008). Resolution of GPS carrier-phase ambiguities in precise point positioning (PPP) with daily observations. Journal of Geodesy, 82(7), 389-399.

Geng, J., Teferle, F. N., Shi, C., Meng, X., Dodson, A. H and Liu, J. (2009). Ambiguity resolution in precise point positioning with hourly data. GPS solutions, 13(4), 263270.

Geng, J., Jiang, P and Liu, J. (2017). Integrating GPS with GLONASS for high - rate seismogeodesy. Geophysical research letters, 44(7), 3139-3146.

Goudarzi, M. A and Banville, S. (2018). Application of PPP with ambiguity resolution in earth surface deformation studies: a case study in eastern Canada. Survey Review, 50(363), 531-544.

Joosten, P. (2000). Fixing the ambiguities-are you sure they're right?. GPS world, 11(5), 46-51.

Lagler, K., Schindelegger, M., Böhm, J., Krásná, H and Nilsson, T. (2013). GPT2: Empirical slant delay model for radio space geodetic techniques. Geophysical research letters, 40(6), 1069-1073.

Li, X., Ge, M., Zhang, X., Zhang, Y., Guo, B., Wang, R ... and Wickert, J. (2013). Real - time high - rate co seismic displacement from ambiguity - fixed precise point positioning: Application to earthquake early warning. Geophysical Research Letters, 40(2), 295-300.

Li, P., Zhang, X., Ren, X., Zuo, X and Pan, Y. (2016). Generating GPS satellite fractional cycle bias for ambiguity-fixed precise point positioning. GPS solutions, 20(4), 771-782.

Li, X., Chen, X., Ge, M and Schuh, H. (2018). Improving multi-GNSS ultra-rapid orbit determination for real-time precise point positioning. Journal of Geodesy, 1-20.

Li, X., Li, X., Liu, G., Feng, G., Guo, F., Yuan, Y and Zhang, K. (2018). Spatial-temporal characteristic of BDS phase delays and PPP ambiguity resolution with 
GEO/IGSO/MEO satellites. GPS Solutions, 22(4), 123.

Pan, L., Cai, C., Santerre, R and Zhu, J. (2014). Combined GPS/GLONASS precise point positioning with fixed GPS ambiguities. Sensors, 14(9), 17530-17547.

Pehlivan, H., Bezcioğlu, M and Yılmaz, M. (2019). PERFORMANCE OF NETWORK RTK CORRECTION TECHNIQUES (FKP, MAC and VRS) UNDER LIMITED SKY VIEW CONDITION. International Journal of Engineering and Geosciences, 4(3), 106-114.

Petit, G.; Luzum, B. IERS Conventions (2010). Bureau International des Poids et Mesures Sevres (France), 2010. Available online: https://www.iers.org/IERS/EN/Publications/TechnicalN otes/tn36

.html (accessed on 1 July 2018).

Teunissen, P. J. G and Khodabandeh, A. (2015). Review and principles of PPP-RTK methods. Journal of Geodesy, 89(3), 217-240

Teunissen, P and Montenbruck, O. (Eds.). (2017). Springer handbook of global navigation satellite systems. Springer.

Tusat, E and Ozyuksel, F. (2018). Comparison of GPS satellite coordinates computed from broadcast and IGS final ephemerides. International Journal of Engineering and Geosciences, 3(1), 12-19.

Schwarz, C. R., Snay, R. A and Soler, T. (2009). Accuracy assessment of the National Geodetic Survey's OPUS-RS utility. GPS solutions, 13(2), 119-132.
Shi, C., Lou, Y. D., Zhang, H. P., Zhao, Q., Geng, J., Wang, R. ... and Liu, J. (2010). Seismic deformation of the Mw 8.0 Wenchuan earthquake from high-rate GPS observations. Advances in Space Research, 46(2), 228235.

Wielgosz, P. (2011). Quality assessment of GPS rapid static positioning with weighted ionospheric parameters in generalized least squares. GPS solutions, 15(2), 89-99.

Wu, J. T., Wu, S. C., Hajj, G. A., Bertiger, W. I and Lichten, S. M. (1992, August). Effects of antenna orientation on GPS carrier phase. In Astrodynamics 1991 (pp. 1647-1660).

Xiao, G., Li, P., Sui, L., Heck, B and Schuh, H. (2019). Estimating and assessing Galileo satellite fractional cycle bias for PPP ambiguity resolution. GPS Solutions, 23(1), 3.

Yigit C.O, Gikas V., Alcay S and Ceylan A. (2014) Performance evaluation of short to long term GPS, GLONASS and GPS/GLONASS post-processed PPP, Survey Review, 46(3), 155-166.

Zumberge, J. F., Heflin, M. B., Jefferson, D. C., Watkins, M. M and Webb, F. H. (1997). Precise point positioning for the efficient and robust analysis of GPS data from large networks. Journal of geophysical research: solid earth, 102(B3), 5005-5017. 